\title{
Reduced basis finite element heterogeneous multiscale method for high-order discretizations of elliptic homogenization problems
}

\author{
Assyr Abdulle, Yun Bai \\ ANMC, Section de Mathématiques, École Polytechnique Fédérale de Lausanne, \\ 1015 Lausanne, Switzerland.
}

\begin{abstract}
A new finite element method for the efficient discretization of elliptic homogenization problems is proposed. These problems, characterized by data varying over a wide range of scales cannot be easily solved by classical numerical methods that need mesh resolution down to the finest scales and multiscale methods capable of capturing the large scale components of the solution on macroscopic meshes are needed. Recently, the finite element heterogeneous multiscale method (FE-HMM) has been proposed for such problems, based on a macroscopic solver with effective data recovered from the solution of micro problems on sampling domains at quadrature points of a macroscopic mesh. Departing from the approach used in the FE-HMM, we show that interpolation techniques based on the reduced basis methodology (an offline-online strategy) allow one to design an efficient numerical method relying only on a small number of accurately computed micro solutions. This new method, called the reduced basis finite element heterogeneous multiscale method (RB-FE-HMM) is significantly more efficient than the FE-HMM for high order macroscopic discretizations and for three-dimensional problems, when the repeated computation of micro problems over the whole computational domain is expensive. A priori error estimates of the RB-FE-HMM are derived. Numerical computations for two and three dimensional problems illustrate the applicability and efficiency of the numerical method.
\end{abstract}

Keywords: heterogeneous multiscale method, reduced basis method, high order finite element method, homogenization problems

2000 MSC: 65N30, 65M12, 65D05, 74Q05, 74Q20

\section{Introduction}

The characterization of effective properties of physical processes in heterogeneous media is a basic problem for many applications. We mention for example the study of thermal diffusion or elastic properties in composite materials. As the heterogeneities of the material occur at microscopic scales, usually much smaller than the scale of interest, it is computationally difficult if not impossible to use a standard numerical method (e.g., as the finite

Email address: assyr.abdulle@epfl.ch, yun.bai@epfl.ch (Assyr Abdulle, Yun Bai) 
element method (FEM), the finite volume method (FVM) or the finite difference method $(\mathrm{FDM}))$ to discretize the physical domain of the composite material going down to the finest scales. A successful theoretical approach for such problems modeled by partial differential equations (PDEs) uses homogenization theory. In this framework, one aims at deriving an effective equation (the homogenized equation) for the PDE under study, where the fine scales have been averaged out [1, 2]. However such a strategy cannot be used directly for numerical computations, as the effective parameters are rarely known in explicit form. This has triggered the development of multiscale methods capable of capturing the coarse behavior of the problem without resolving the full fine scale details on the whole computational domain. Among the huge literature available we mention [3, 4, 5, 6, 7] and references therein.

Here we focus on the finite element heterogeneous multiscale method (FE-HMM), a numerical homogenization method developed in the framework of the heterogeneous multiscale method (HMM) proposed in [8]. In a nutshell, these methods rely on a macro partition of the computational domain with effective (homogenized) coefficients recovered (on the fly) from micro problems defined on sampling domains located at quadrature points within each element of the macro partition (see [9] for a recent review of the FE-HMM). As macro and micro meshes have to be refined simultaneously for optimal convergence rates -as shown by the fully discrete error analysis [10]- such methods require a large number of repeated micro computations. This is a common feature shared by any numerical homogenization method. For higher order macro methods (where more sampling domains are required) or three-dimensional problems, these methods can become computationally very expensive (even though order of magnitude cheaper than a full fine scale approach). Attempts to reduce the computational cost have been pursued in [11], where fast micro solvers have been coupled with standard FEM. By selecting a special quadrature formula with integration points on the interfaces of the macro partition, one can also in some situations reduce the computational cost (this does however only reduce the constant in front of the computational cost for the FE-HMM, e.g., a reduction factor of one half is reported in [12] for two dimensional problems with first or second order macro solvers). Finally, by using adaptive strategies, it has been shown in [13, 14] that substantial computational savings can be achieved as new micro computations are only required in elements marked for refinement.

In this paper we depart from the classical approach of the FE-HMM which consists in solving a micro problem on a sampling domain at each quadrature point of a macro FEM with numerical integration. In our new approach, a small number of sampling domains located anywhere in the computational domain are computed accurately in an offline stage, following the methodology of reduced basis (RB). A suitable interpolation of these precomputed microsolutions is used in an online stage to compute the macro solution in a cheap and efficient way. We demonstrate that such a strategy allows to design efficient

- numerical homogenization methods with high order macro solvers;

- numerical homogenization methods for three-dimensonal problems.

RB techniques for model reduction, pioneered in [15, 16, 17], have seen recently a renewed interest thanks to the development of new sampling techniques and rigorous a posteriori er- 
ror bounds for outputs of interest [18] (see also [19, 20] for additional references on the recent literature). In the context of numerical homogenization, the use of RB was first proposed in [21, 22]. We also note that in [23], the RB techniques were used in combination with the multiscale finite element method [3]. While the emphasis in [21, 22] was on parametrizing various configurations of cell problems (e.g. inclusion with various shapes, etc.), here, building on [21, 22, we focus on integrating the RB methodology in a micro macro FEM such as the FE-HMM. As in the FE-HMM, we compute a macro FEM based on quadrature formula (QF), but do not solve micro FE problems around every integration point of the QF in each macro element. Instead, we select by a greedy procedure a number of representative sampling domains on which we solve accurate micro problems. Their corresponding solutions span the $\mathrm{RB}$ space. This procedure is called the offline stage, in the RB terminology, and is usually only done once, as a pre-processing step. In a so-called online stage, the effective solution is obtained from the macro solver of the FE-HMM with effective coefficients recovered from micro problems solved in the RB space. The required data at the macro integration points are now obtained from the solutions of small dimensional linear problems involving suitable interpolations of the precomputed RB space. Unlike the FE-HMM, in the RB-FE-HMM the dimension of the micro linear systems solved in the online stage is independent of the macro resolution (e.g., macro meshsize). In turn, there is no need in the RB-FE-HMM to refine simultaneously macro and micro meshes to obtain optimal convergence rates. Thus, expensive micro FE computations as required by the FE-HMM are avoided. High order macroscopic methods can be designed with the same set of RB as used for linear macro FE. As illustrated in numerical experiments, the RB-FE-HMM is particularly suited for high order macro FEM or high dimensional problems for which the cost of the standard FE-HMM justifies (even when a single macroscopic output is required) the overhead of the offline work in the RB methodology.

A priori error analysis including macro error, micro error, resonance error and error coming from the use of the RB is derived. We also show how $\mathrm{RB}$ can be used to reconstruct fine scale information on the whole computational domain to approximate the fine scale solution. Such a reconstruction is simpler than the similar procedure for the FE-HMM. Indeed, we do not need to store micro solutions in each sampling domains, nor to implement a periodic extension of the micro solutions in each macro elements, which might be cumbersome, specially for three dimensional problems with simplicial elements.

Our paper is organized as follows. In section 2, we introduce the homogenization problem and give a short description of the FE-HMM. Section 3 is devoted to our multiscale method, the RB-FE-HMM. The a priori error analysis of the method is presented in Section 4. Finally, various numerical examples in two and three dimensions are presented in Section 5, to illustrate the behavior and the efficiency of the proposed method.

Notations Let $\Omega \subset \mathbb{R}^{d}$ be an open set, and $Y=\left(-\frac{1}{2}, \frac{1}{2}\right)^{d}$. Denote the standard Sobolev Space by $W^{\ell, p}(\Omega)$. For $p=2$, we sometimes use the notation $W^{\ell, p}(\Omega)=\mathcal{H}^{\ell}(\Omega)$. $\mathcal{H}_{0}^{1}(\Omega)$ denotes the closure of $C_{0}^{\infty}(\Omega)$ in $\mathcal{H}^{1}(\Omega)$. We define $\mathcal{H}_{\text {per }}^{1}(Y):=\left\{g \in \mathcal{H}^{1}(Y) \mid g\right.$ periodic in $\left.Y\right\}$. We also have the property that $\mathcal{H}_{p e r}^{1}(Y)=\overline{C_{p e r}^{\infty}(Y)}$, where $C_{p e r}^{\infty}(Y)$ is a subspace of $C^{\infty}\left(\mathbb{R}^{d}\right)$ of periodic functions in the cube $Y$. The Frobenius norm of a matrix, denoted by $\|\cdot\|_{\mathcal{F}}$, is 
defined as $\|A\|_{\mathcal{F}}=\sqrt{\operatorname{trace}\left(A^{T} A\right)}$.

\section{Model problem, homogenization and FE-HMM}

Let $\Omega$ be a bounded polyhedron in $\mathbb{R}^{d}$ with $d \leq 3$. We consider a second-order elliptic problems of the form

$$
\begin{array}{rlrl}
-\nabla \cdot\left(a^{\varepsilon}(x) \nabla u^{\varepsilon}(x)\right) & =f, & \text { in } \Omega \\
u^{\varepsilon}(x) & =0, & & \text { on } \partial \Omega,
\end{array}
$$

where $f \in \mathcal{H}^{-1}(\Omega)$. Here, we choose a zero Dirichlet boundary condition for simplicity. We emphasize that the numerical method presented below can be readily generalized to other boundary conditions, e.g.,

$$
\begin{aligned}
u^{\varepsilon}(x) & =g_{D}(x), & & \text { on } \partial \Omega_{D} \\
n \cdot\left(a^{\varepsilon}(x) \nabla u^{\varepsilon}(x)\right) & =g_{N}(x), & & \text { on } \partial \Omega_{N},
\end{aligned}
$$

where $\partial \Omega=\partial \Omega_{D} \cup \partial \Omega_{N}$, with $\partial \Omega_{D}$ having non-zero measure, and $g_{D} \in \mathcal{H}^{\frac{1}{2}}\left(\partial \Omega_{D}\right), g_{N} \in$ $\mathcal{H}^{-\frac{1}{2}}\left(\partial \Omega_{N}\right)$. The $d \times d$ tensor $a^{\varepsilon}(x)$ is assumed to be uniformly elliptic and bounded, i.e.,

$$
\lambda|\xi|^{2} \leq a^{\varepsilon}(x) \xi \cdot \xi,\left|a^{\varepsilon}(x) \xi\right| \leq \Lambda|\xi|, \forall \xi \in \mathbb{R}^{d}, \forall \varepsilon>0
$$

for a.e $x \in \Omega$. The tensor is allowed to vary on a small spatial scale denoted by $\varepsilon$. The behavior of $a^{\varepsilon}$ makes a standard numerical approximation such as FEM, FVM or FDM very costly if not impossible. Therefore, an upscaling procedure is required. The theoretical foundation of this procedure usually relies on homogenization theory that we briefly review for completeness. For simplicity of the presentation, we will assume that the tensor $a^{\varepsilon}$ is symmetric. We emphasize that both the FE-HMM and the RB-FE-HMM can be extended to non-symmetric problems (for the RB-FE-HMM, this requires the introduction of a dual problem for the approximation space).

\subsection{Homogenization}

Mathematical homogenization is concerned with the study of PDEs with rapidly oscillating coefficients varying over multiple spatial or temporal scales and aims at describing "averaged" (i.e., homogenized) solutions of the original PDEs. In the periodic case, i.e., when $a^{\varepsilon}(x)=a(x / \varepsilon)=a(y)$ is $Y$-periodic in $y$, a formal approach relies on the multiple scale expansion. For a function $\phi(x, y)$, where $x \in \Omega$ is called the slow variable and $y=x / \varepsilon \in Y$ (the domain of periodicity, e.g., $\left.Y=\left(-\frac{1}{2}, \frac{1}{2}\right)^{d}\right)$ is called the fast variable, we consider $\phi(x, x / \varepsilon)$. We then look for a solution $u^{\varepsilon}$ of (2.1) in the form $u^{\varepsilon}(x)=u^{0}(x, x / \varepsilon)+\varepsilon u^{1}(x, x / \varepsilon)+\varepsilon^{2} u^{2}(x, x / \varepsilon)+\ldots$. Upon inserting this expression in (2.1) and identifying the power of $\varepsilon$, one obtains (formally) that $u^{0}(x, x / \varepsilon)=u^{0}(x)$, where $u^{0}(x)$ is a solution of the following "homogenized equation" [1]

$$
\begin{aligned}
-\nabla \cdot\left(a^{0} \nabla u^{0}(x)\right) & =f(x) \text { in } \Omega . \\
u^{0}(x) & =0 \text { on } \partial \Omega .
\end{aligned}
$$


In the above situation, the homogenized tensor $a^{0}$ is constant and is given by a suitable average of $d$ solutions of a boundary value problem in the domain $Y$ (with $d$ different right hand-side). This boundary value problem is usually called the cell problem. It can be shown that $a^{0}$ it is again elliptic. These formal computations do not provide a converge result of $u^{\varepsilon}$ towards $u^{0}$. Using Tartar's method of oscillating test functions [24] (see also [1]) it is possible to show that $u^{\varepsilon} \rightarrow u^{0}$ weakly in $\mathcal{H}_{0}^{1}(\Omega), a^{\varepsilon} \nabla u^{\varepsilon} \rightarrow a^{0} \nabla u^{0}$ weakly in $\left(L^{2}(\Omega)\right)^{d}$, where $u^{0}$ is the solution of 2.3 .

The problem gets more involved if $a^{\varepsilon}$ is not periodic. On the theoretical side, one can rely on $H$-convergence [24] which ensures the existence of a subsequence of the matrices $a^{\varepsilon}$ and a homogenized tensor $a^{0}$ (again uniformly elliptic and bounded) such that for the corresponding subsequence, $u^{\varepsilon}$ and $a^{\varepsilon} \nabla u^{\varepsilon}$ enjoy the same convergence properties as described above for the periodic case. However for non-periodic oscillating tensors, the homogenized tensors are in general not constant, i.e., $a^{0}=a^{0}(x)$ and usually unknown (in an explicit analytical form). For numerical homogenization one usually assumes scale separation between fast and slow variables and relies on local problems to compute the homogenized tensor for a given value of $x \in \Omega$. A typical example is when the tensor is locally periodic, i.e., $a^{\varepsilon}(x)=a(x, x / \varepsilon)=a(x, y)$ is $Y$ periodic in the $y$ variable. Even in such a simplified situation, we have an infinite number of cell problems (for each $x \in \Omega$ ), whose solutions must usually be computed numerically. The task in numerical homogenization is thus to design an algorithm capable of computing an approximation of the homogenized solution $u^{0}(x)$, relying on a finite number of cell problems chosen in such a way that the overall computation is efficient and reliable.

\subsection{The FE-HMM}

The FE-HMM belongs to the class of numerical homogenization methods described in the previous subsection. It is based on a macroscopic FEM with QF defined on a macroscopic partition of the domain $\Omega$. As $a^{0}(x)$ is usually unknown, the method is supplemented by microscopic FEMs defined on sampling domains centred at the macroscopic quadrature points of the QF, relying only on the data given in (2.1). A suitable averaging of the solutions of the microscopic FEMs allows to recover the missing macroscopic tensor at the macroscopic quadrature points.

The method is applicable to general problems and does not rely on a specific structure of the oscillating tensor such as periodicity. We however assume that there is a well defined homogenized problem with certain smoothness properties and a scale separation between fast and slow variables, which we will make precise in the following. The FE-HMM is based on a macro finite element (FE) space

$$
S_{0}^{\ell}\left(\Omega, \mathcal{T}_{H}\right)=\left\{v^{H} \in \mathcal{H}_{0}^{1}(\Omega) ;\left.v^{H}\right|_{K} \in \mathcal{R}^{\ell}(K), \forall K \in \mathcal{T}_{H}\right\}
$$

where $\mathcal{T}_{H}$ is a family of (macro) partition of $\Omega$ in simplicial or quadrilateral elements $K$ of diameter $H_{K}$, and $\mathcal{R}^{\ell}(K)$ is the space $\mathcal{P}^{\ell}(K)$ of polynomials on $K$ of total degree at most $\ell$ if $K$ is a simplicial FE, or the space $\mathcal{Q}^{\ell}(K)$ of polynomials on $K$ of degree at most $\ell$ in each variable if $K$ is a quadrilateral FE. For a given macro partition, we define as usual 
$H:=\max _{K \in \mathcal{T}_{H}} H_{K}$. We note that $H$ in our discretization is allowed to be much larger than $\varepsilon$.

Defining a $\mathrm{QF}\left\{\hat{x}_{j}, \hat{\omega}_{j}\right\}_{j=1}^{J}$ on a reference element $\hat{K}$, we equip each element $K$ with a corresponding QF $\left\{x_{K_{j}}, \omega_{K_{j}}\right\}_{j=1}^{J}$ by using a $C^{1}$-diffeomorphism. We will make the following assumptions on the quadrature formula (similar to the requirement for standard FEM with numerical quadrature [25])

(Q1) $\hat{\omega}_{j}>0, j=1, \ldots, J, \quad \sum_{j=1}^{J} \hat{\omega}_{j}\left|\nabla \hat{p}\left(\hat{x}_{j}\right)\right|^{2} \geq \hat{\lambda}\|\nabla \hat{p}\|_{L^{2}(\hat{K})}^{2}, \forall \hat{p}(\hat{x}) \in \mathcal{R}^{\ell}(\hat{K})$, with $\hat{\lambda}>0$

(Q2) $\int_{\hat{K}} \hat{p}(\hat{x}) d \hat{x}=\sum_{j \in J} \hat{\omega}_{j} \hat{p}\left(\hat{x}_{j}\right), \forall \hat{p}(\hat{x}) \in \mathcal{R}^{\sigma}(\hat{K})$, where $\sigma=\max (2 \ell-2, \ell)$ if $\hat{K}$ is a simplicial $\mathrm{FE}$, or $\sigma=\max (2 \ell-1, \ell+1)$ if $\hat{K}$ is a rectangular $\mathrm{FE}$.

For each macro element $K \in \mathcal{T}_{H}$ and each integration point $x_{K_{j}} \in K, j=1, \ldots, J$, we define the sampling domains $K_{\delta_{j}}=x_{K_{j}}+(-\delta / 2, \delta / 2)^{d}$, $(\delta \geq \varepsilon)$. For a sampling domain $K_{\delta_{j}}$, we then define a micro FE space $S^{q}\left(K_{\delta_{j}}, \mathcal{T}_{h}\right) \subset W\left(K_{\delta_{j}}\right)$ with simplicial or quadrilateral FEs and piecewise polynomial of degree $q\left(\mathcal{T}_{h}\right.$ is a conformal and shape regular family of triangulation). The space $W\left(K_{\delta_{j}}\right)$ is either the Sobolev space

$$
W\left(K_{\delta_{j}}\right)=\mathcal{W}_{p e r}^{1}\left(K_{\delta_{j}}\right)=\left\{z \in \mathcal{H}_{p e r}^{1}\left(K_{\delta_{j}}\right) ; \int_{K_{\delta_{j}}} z d x=0\right\}
$$

for a periodic coupling or

$$
W\left(K_{\delta_{j}}\right)=\mathcal{H}_{0}^{1}\left(K_{\delta_{j}}\right)
$$

for a coupling with Dirichlet boundary conditions.

FE-HMM. At the macroscopic level, the numerical method is defined as follows: find $u^{H} \in S_{0}^{\ell}\left(\Omega, \mathcal{T}_{H}\right)$ such that

$$
B_{H}\left(u^{H}, v^{H}\right)=\int_{\Omega} f v^{H} d x, \forall v^{H} \in S_{0}^{\ell}\left(\Omega, \mathcal{T}_{H}\right),
$$

where

$$
B_{H}\left(v^{H}, w^{H}\right):=\sum_{K \in \mathcal{T}_{H}} \sum_{j=1}^{J} \frac{\omega_{K_{j}}}{\left|K_{\delta_{j}}\right|} \int_{K_{\delta_{j}}} a^{\varepsilon}(x) \nabla v_{K_{j}}^{h}(x) \cdot \nabla w_{K_{j}}^{h}(x) d x .
$$

In (2.7) $v_{K_{j}}^{h}$ (respectively $w_{K_{j}}^{h}$ ) denotes the solution of the following micro problem: find $v_{K_{j}}^{h}$ such that $v_{K_{j}}^{h}-v_{\text {lin,j }}^{H}(x) \in S^{q}\left(K_{\delta_{j}}, \mathcal{T}_{h}\right)$ and

$$
\int_{K_{\delta_{j}}} a^{\varepsilon}(x) \nabla v_{K_{j}}^{h}(x) \cdot \nabla z^{h}(x) d x=0 \quad \forall z^{h} \in S^{q}\left(K_{\delta_{j}}, \mathcal{T}_{h}\right),
$$

where we used the notation $v_{l i n, j}^{H}(x):=v^{H}\left(x_{K_{j}}\right)+\left(x-x_{K_{j}}\right) \cdot \nabla v^{H}\left(x_{K_{j}}\right)$. 
A priori estimates. Coercivity and boundedness of $B_{H}(\cdot, \cdot)$ can be shown provided ellipticity and boundedness of $a^{\varepsilon} 1$. The following convergence results have been derived in [10, 26]

$$
\left\|u^{0}-u^{H}\right\|_{\mathcal{H}^{1}(\Omega)} \leq C\left(H^{\ell}+r_{H M M}\right),\left\|u^{0}-u^{H}\right\|_{L^{2}(\Omega)} \leq C\left(H^{\ell+1}+r_{H M M}\right),
$$

where we assume the regularity $u^{0} \in \mathcal{H}^{\ell+1}(\Omega)$. Notice that the constants $C$ in the above estimates are independent of $H, h, \varepsilon$. Optimal convergence rates for the macro FEM can be observed in the above error bounds. The term $r_{H M M}$ can be further decomposed into two parts, $r_{H M M}=r_{M O D}+r_{M I C}$. The term $r_{M O D}$ (called modeling error) quantifies the error induced by artificial micro boundary conditions or non-optimal sampling of the micro structure. It does not depend on the macro or micro meshsizes and can be analyzed for locally periodic tensor [26, 27, 12]. The term $r_{M I C}$ (called micro error) quantifies the error due to the micro FEM. Assuming appropriate regularity and bounds on $a^{\varepsilon}, r_{M I C}$ can be bounded in terms of the micro meshize as

$$
r_{M I C} \leq C\left(\frac{h}{\varepsilon}\right)^{2 q} .
$$

Notice that in the estimate $2.10, \mathcal{H}^{q+1}$ regularity of the exact solution of the cell problem (2.8) has been assumed. For $q=1$, the estimate of the micro error $r_{M I C}$ was first presented in [10], generalized to high order in [28, Corollary 10] (see also [9, Lemma 10] and [29]).

Remark 2.1. If $\left.a^{\varepsilon}\right|_{K} \in W^{1, \infty}(K), \forall K \in \mathcal{T}_{H}$ and $\left|a_{i j}^{\varepsilon}\right|_{W^{1, \infty}(K)} \leq C_{K^{\varepsilon}}{ }^{-1}$ for $i, j=1, \ldots d$, then 2.10 holds for $q=1$ for arbitrary $K_{\delta_{j}}$ if $W\left(K_{\delta_{j}}\right)=\mathcal{H}_{0}^{1}\left(K_{\delta_{j}}\right)$. If $a^{\varepsilon}=a(x, x / \varepsilon)=$ $a(x, y)$ is $Y$-periodic with respect to the $y$ variable (locally periodic tensor) and if $K_{\delta_{j}}$ covers an integer number of periods, then (2.10) holds for higher order q provided $W\left(K_{\delta_{j}}\right)=$ $\mathcal{W}_{\text {per }}^{1}\left(K_{\delta_{j}}\right)$ and $a^{\varepsilon}=a(\cdot, y)$ is smooth enough (see [28, Remark 5.1 and Corollary 10] or [29, Remark 4$]$ for details).

Complexity and optimal macro-micro refinement. Taking $N_{\text {mic }}$ elements in each space dimension for the discretization of the sampling domain $K_{\delta_{j}}$, we have $h=\delta / N_{\text {mic }}$ and thus $\hat{h}=(\delta / \varepsilon) \cdot\left(1 / N_{m i c}\right)$. Since $\delta$ scales with $\varepsilon$, typically $\delta=C \varepsilon$ (where $C$ is a constant of moderate size), we obtain $\hat{h}=\left(C / N_{\text {mic }}\right)$, independent of $\varepsilon$.

We denote by $M_{m i c}=\mathcal{O}\left(\hat{h}^{-d}\right)$ the number of degrees of freedom (DOF) for the micro FEM and by $M_{m a c}$, the number of DOF of the macro FEM. For quasi-uniform macro meshes, the macro meshsize $H$ and the micro meshsize $\hat{h}$ are related to $M_{m a c}$ and $M_{m i c}$ as

$$
H=\mathcal{O}\left(M_{\text {mac }}^{-1 / d}\right), \quad \hat{h}=\mathcal{O}\left(M_{m i c}^{-1 / d}\right) .
$$

In view of (2.9) and 2.10), optimal macroscopic convergence rates (up to a modeling error $r_{M O D}$ independ of $\left.H, h\right)$ are obtained for quasi-uniform microscopic meshsizes given by

$$
\hat{h} \simeq H^{\frac{\ell}{2 q}} \quad \text { for the } \mathcal{H}^{1} \text { norm, } \quad \hat{h} \simeq H^{\frac{\ell+1}{2 q}} \text { for the } L^{2} \text { norm. }
$$

\footnotetext{
${ }^{1}$ Notice that the regularity $\|v\|_{\mathcal{H}^{2}(\Omega)} \leq C\left\|L^{*} v\right\|_{L^{2}(\Omega)}, \forall v \in \mathcal{H}^{2}(\Omega) \cap \mathcal{H}_{0}^{1}(\Omega)$, where $L^{*}$ is the adjoint operator of $L=-\nabla \cdot\left(a^{0}(x) \nabla\right)$, is needed for optimal $L^{2}$ estimates [25].
} 
The corresponding complexity in term of macro DOF reads

$$
\begin{aligned}
& \underbrace{H^{-d}}_{M_{m a c}} \cdot \underbrace{H^{\frac{-d \ell}{2 q}}}_{M_{m i c}} \cdot n_{s}=\left(M_{m a c}\right)^{1+\frac{\ell}{2 q}} \cdot n_{s} \quad \text { for the } \mathcal{H}^{1} \text { norm, } \\
& \underbrace{H^{-d}}_{M_{m a c}} \cdot \underbrace{H^{\frac{-d(\ell+1)}{2 q}}}_{M_{m i c}} \cdot n_{s}=\left(M_{m a c}\right)^{1+\frac{\ell+1}{2 q}} \cdot n_{s} \quad \text { for the } L^{2} \text { norm, }
\end{aligned}
$$

where $n_{s}$ denotes the number of sampling domains per macro element $K \in \mathcal{T}_{H}$. As can be seen above and as first noticed in [10] the overall complexity of the method is a function of $M_{m a c}$ and $M_{m i c}$ and in general superlinear with respect to the macro DOF. For example, using piecewise linear polynomials on simplicial FEs, assuming quasi-uniform macro and micro meshes and that the complexity is proportional to the total DOF we obtain a cost of $\mathcal{O}\left(M_{\text {mac }}^{3 / 2}\right)$ $\left(\mathcal{H}^{1}\right.$ norm $)$ and $\mathcal{O}\left(M_{\text {mac }}^{2}\right)\left(L^{2}\right.$ norm $) .2$ In contrast, the memory demand is proportional to $M_{m a c}+M_{m i c}$ only as the micro problem, being independent of one another, can be solved one at a time. Finally we note that by using spectral methods or $p-F E M$ for the micro solvers can reduce the complexity of the overall FE-HMM (up to log-linear complexity). This was investigated in [11]. Such an approach requires however high regularity in the oscillating tensor $a^{\varepsilon}$ which may not hold for some applications as for example in material science.

\section{Reduced Basis FE-HMM (RB-FE-HMM)}

As can be seen from the discussion in the previous section, the main cost of the FE-HMM comes from the computation of the cell problems, whose number and DOF increase as we refine the macro mesh for an appropriate approximation of the homogenized solution. In this section we explain how RB can be coupled to the FE-HMM to drastically reduce the cost of solving repeatedly a large number of cell problems.

\subsection{Parametrized micro problems and numerical homogenized tensor}

In what follows, it will be convenient to denote the micro FE space by $S^{q}\left(K_{\delta_{j}}, \mathcal{N}\right)$ instead of $S^{q}\left(K_{\delta_{j}}, \mathcal{T}_{h}\right)$ to emphasize on the dimension $\mathcal{N}$ of the micro FE space. Likewise, the micro function $v_{K_{j}}^{h}$, the solution of problem 2.8 will be denoted by $v_{\mathcal{N}, K_{j}}$. We first notice that $v_{\mathcal{N}, K_{j}}$ can be decomposed as

$$
v_{\mathcal{N}, K_{j}}(x)=v_{\text {lin,j }}^{H}(x)+\sum_{i=1}^{d} \chi_{\mathcal{N}, K_{j}}^{i}(x) \frac{\partial v_{l i n, j}^{H}}{\partial x_{i}}
$$

where $\chi_{\mathcal{N}, K_{j}}^{i}(x), i=1, \ldots, d$ are solutions of

$$
\int_{K_{\delta_{j}}} a^{\varepsilon}(x) \nabla \chi_{\mathcal{N}, K_{j}}^{i}(x) \cdot \nabla z_{\mathcal{N}}(x) d x=-\int_{K_{\delta_{j}}} a^{\varepsilon}(x) \mathbf{e}_{\mathbf{i}} \cdot \nabla z_{\mathcal{N}}(x) d x \quad \forall z_{\mathcal{N}} \in S^{q}\left(K_{\delta_{j}}, \mathcal{N}\right) .
$$

\footnotetext{
2 Notice that as the micro problems are solved independently, the method is well suited for parallel implementation which can reduce significantly the complexity of the FE-HMM.
} 
In the above formula, the vector $\mathbf{e}_{\mathbf{i}}, i=1 \ldots d$ denote the canonical basis of $\mathbb{R}^{d}$. We now map a sampling domain $K_{\delta_{j}}$ in the reference domain $Y$ through $x=G_{x_{K_{j}}}(y)=x_{K_{j}}+\delta y$ and consider $\hat{\chi}_{\mathcal{N}, K_{j}}^{i}$ the solution of

$$
\begin{aligned}
b\left(\hat{\chi}_{\mathcal{N}, K_{j}}^{i}, \hat{z}_{\mathcal{N}}\right) & :=\int_{Y} a_{x_{K_{j}}}(y) \nabla \hat{\chi}_{\mathcal{N}, K_{j}}^{i}(y) \cdot \nabla \hat{z}_{\mathcal{N}}(y) d y \\
& =-\int_{Y} a_{x_{K_{j}}}(y) \mathbf{e}_{\mathbf{i}} \cdot \nabla \hat{z}_{\mathcal{N}}(y) d y=: l_{i}\left(\hat{z}_{\mathcal{N}}\right) \quad \forall \hat{z}_{\mathcal{N}} \in S^{q}(Y, \mathcal{N}),
\end{aligned}
$$

where we note that $a^{\varepsilon}\left(G_{x_{K_{j}}}(y)\right)$ can be parametrized by $x_{K_{j}} \in \Omega$ and we therefore use the notation $a_{x_{K_{j}}}(y):=a^{\varepsilon}\left(G_{x_{K_{j}}}(y)\right)$. The FE space $S^{q}(Y, \mathcal{N})$ has a triangulation $\mathcal{T}_{\hat{h}}$ with $\mathcal{N}=\mathcal{O}\left(\hat{h}^{-d}\right)$ denoting its degrees of freedom. Functions in $S^{q}(Y, \mathcal{N})$ will have a subscript $\mathcal{N}\left(\right.$ e.g., $\left.\hat{z}_{\mathcal{N}}\right)$. It is easily seen that

$$
v_{\mathcal{N}, K_{j}}=v_{\text {lin }, j}^{H}(x)+\delta \sum_{i=1}^{d} \hat{\chi}_{\mathcal{N}, K_{j}}^{i}\left(G_{x_{K_{j}}}^{-1}(x)\right) \frac{\partial v_{\text {lin,j }}^{H}}{\partial x_{i}} .
$$

The following reformulation of the FE-HMM makes a link between the micro problems and the effective tensor obtained by the above micro-macro procedure. We have [28, Lemma $5.4],[29]$

$$
\frac{1}{\left|K_{\delta_{j}}\right|} \int_{K_{\delta_{j}}} a^{\varepsilon}(x) \nabla v_{\mathcal{N}, K_{j}}(x) \cdot \nabla w_{\mathcal{N}, K_{j}}(x) d x=a_{\mathcal{N}}^{0}\left(x_{K_{j}}\right) \nabla v^{H}\left(x_{K_{j}}\right) \cdot \nabla w^{H}\left(x_{K_{j}}\right) .
$$

Inserting (3.15) in 2.7) we obtain

$$
B_{H}\left(v^{H}, w^{H}\right):=\sum_{K \in \mathcal{T}_{H}} \sum_{j=1}^{J} \omega_{K_{j}} a_{\mathcal{N}}^{0}\left(x_{K_{j}}\right) \nabla v_{\text {lin,j }}^{H}\left(x_{K_{j}}\right) \cdot \nabla w^{H}\left(x_{K_{j}}\right),
$$

where

$$
\left(a_{\mathcal{N}}^{0}\left(x_{K_{j}}\right)\right)_{i k}=\int_{Y} a_{x_{K_{j}}}(y)\left(\nabla \hat{\chi}_{\mathcal{N}, K_{j}}^{i}(y)+\mathbf{e}_{\mathbf{i}}\right) \cdot\left(\nabla \hat{\chi}_{\mathcal{N}, K_{j}}^{k}(y)+\mathbf{e}_{\mathbf{k}}\right) d y
$$

and $\hat{\chi}_{\mathcal{N}, K_{j}}^{i}, \hat{\chi}_{\mathcal{N}, K_{j}}^{k}$ are the solutions of 3.13 .

Remark 3.1. Similar to (3.16), we have the following bilinear form

$$
B_{H}\left(v^{H}, w^{H}\right):=\sum_{K \in \mathcal{T}_{H}} \sum_{j=1}^{J} \omega_{K_{j}} \bar{a}^{0}\left(x_{K_{j}}\right) \nabla v^{H}\left(x_{K_{j}}\right) \cdot \nabla w^{H}\left(x_{K_{j}}\right),
$$

where $\bar{a}^{0}$ is obtained from (3.17), assuming the solutions of problem (3.12) are computed exactly. For the analysis in Section 4 (also for (2.9) ) we also need the auxiliary problem 
corresponding to the FE discretization with numerical quadrature of the homogenized problem (2.3), i.e., the solution $u^{0, H}$ of the following problem

$$
B_{0, H}\left(u^{0, H}, v^{H}\right)=\int_{\Omega} f v^{H} d x, \forall v^{H} \in S_{0}^{\ell}\left(\Omega, \mathcal{T}_{H}\right),
$$

where for $v^{H}, w^{H} \in S_{0}^{\ell}\left(\Omega, \mathcal{T}_{H}\right)$

$$
B_{0, H}\left(v^{H}, w^{H}\right):=\sum_{K \in \mathcal{T}_{H}} \sum_{j=1}^{J} \omega_{K_{j}} a^{0}\left(x_{K_{j}}\right) \nabla v^{H}\left(x_{K_{j}}\right) \cdot \nabla w^{H}\left(x_{K_{j}}\right),
$$

and $a^{0}\left(x_{K_{j}}\right)$ is the homogenized tensor of problem (2.3) evalutaed at the quadrature point $x_{K_{j}}$.

\subsection{Model reduction}

Inspired by the parametrization of the solutions of the micro problems $(3.13)$ in the reference domain, we now describe a model reduction strategy for micro functions used in the FE-HMM. The overall idea is the following. Instead of computing micro functions in each macro elements at the quadrature points, we identify a small number $N$ of carefully precomputed micro functions (to construct the RB space), whose supports can be chosen in the whole computational domain as sketched in Fig.1 (offline stage).

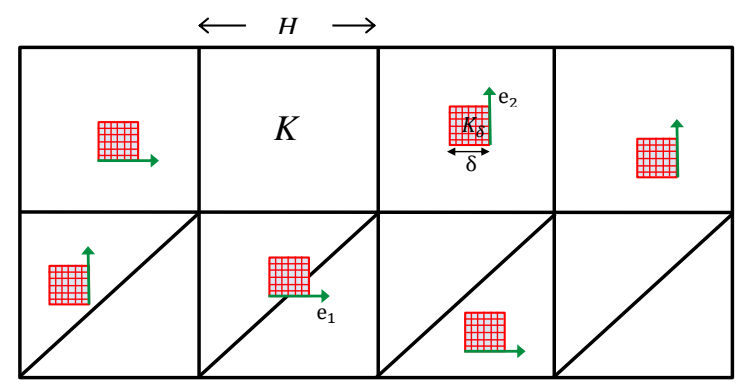

Fig. 1: The supports of the RB functions.

In the online stage, the solution of cell problems at the given quadrature points of the macro elements are then computed in the RB space. No mesh, neither stiffness matrix assembly is needed for these later problems which require only the solution of small linear systems of size $N \times N$. Let $T_{\delta}=x_{\tau}+(-\delta / 2, \delta / 2)^{d}$ be a sampling domain centered at $x_{\tau} \in \Omega$, chosen such that $T_{\delta} \subset \Omega$. For $\left\{\left(T_{\delta}, \mathbf{e}_{\eta}\right) ; T_{\delta} \subset \Omega, \eta=1, \ldots, d\right\}$, we introduce the space of "cell solutions",

$$
\mathcal{M}^{\mathcal{N}}(Y):=\left\{\hat{\xi}_{\mathcal{N}, T_{\delta}}^{\eta} ; T_{\delta} \subset \Omega, \eta=1, \ldots, d\right\}
$$


where $\hat{\xi}_{\mathcal{N}, T_{\delta}}^{\eta}(\cdot): Y \rightarrow \mathbb{R}$ are the solutions of 3.13 associated with the mapping $G_{x_{\tau}}$, i.e., with a tensor $a_{x_{\tau}}(y)=a^{\varepsilon}\left(G_{x_{\tau}}(y)\right)$ and with right-hand side $l_{\eta}(\cdot)$. The functions $\hat{\xi}_{\mathcal{N}, T_{\delta}}^{\eta}$ are computed very accurately. The DOF $\mathcal{N}$ of the FE space $S^{q}(Y, \mathcal{N})$ is thus assumed to be large.

Affine representation of the tensor. A suitable representation of the tensor $a_{x_{\tau}}(y)$ is crucial for the efficiency of the RB method (more precisely, we look for an affine parametrization). The simplest case is when $a_{x_{\tau}}(y)$ is directly available in an affine form

$$
a_{x_{\tau}}(y)=\sum_{q=1}^{Q} \Theta_{q}\left(x_{\tau}\right) a_{q}(y), \forall y \in Y
$$

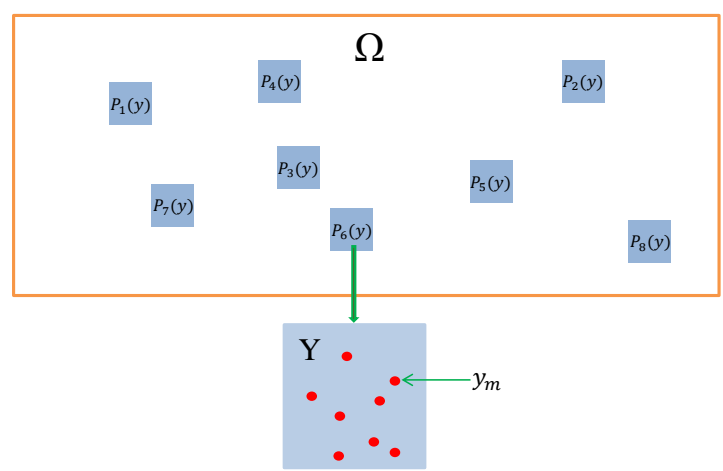

Fig. 2: The EIM basis functions $\left\{P_{q}(y)\right\}_{q=1}^{8}$ and the interpolation points $\left\{y_{m}\right\}_{m=1}^{8}$ on the reference sampling domain $Y$.

If a representation as (3.22) is not available, a greedy algorithm, called the empirical interpolation method (EIM) [30], can be applied to obtain an affine approximation of $a_{x_{\tau}}(y)$ in the form

$$
a_{x_{\tau}}^{M}(y)=\sum_{q=1}^{M} \varphi_{q}\left(x_{\tau}\right) p_{q}(y) .
$$

The idea is to approximate the function $a_{x_{\tau}}^{M}(y)$ by linear combination of "snapshot" $\left\{p_{x_{\tau_{1}}}(y), \ldots\right.$, $\left.p_{x_{\tau_{M}}(y)}\right\}$. For an arbitrary $x_{\tau}$ the linear combination to approximate $a_{x_{\tau}}^{M}(y)$ will be based on interpolation points $y_{1}, \ldots, y_{M}$ in $Y$ (see Figure 2). The space of snapshots, called $S_{M}^{E I M}=\operatorname{span}\left\{p_{q}(y), q=1, \cdots, M\right\}$ and the interpolation points $\left\{y_{m}\right\}_{m=1}^{M}$ are computed in an offline stage with the help of a greedy algorithm controlled by available a posteriori error estimates. In the online stage for a given $a_{x_{\tau}}(y)$ compute (3.23) as follows

- evaluate $a_{x_{\tau}}\left(y_{m}\right)$ at the interpolation points $\left\{y_{m}\right\}_{m=1}^{M}$; 
- solve the interpolation problem (a $M \times M$ linear system)

$$
\sum_{q=1}^{M} p_{q}\left(y_{m}\right) \varphi_{q}\left(x_{\tau}\right)=a_{x_{\tau}}\left(y_{m}\right), m=1, \ldots, M
$$

to find $\varphi_{q}\left(x_{\tau}\right)_{q=1}^{M}$.

Details of the above procedure can be found in [30, 31]. We refer to Section 5 for numerical computations with affine and nonaffine multiscale tensors.

A posteriori error estimator. Crucial for the selection of the reduced basis functions is an appropriate a posteriori error estimator. For a given sampling domain $T_{\delta} \subset \Omega$ let $\xi_{\mathcal{N}, T_{\delta}}^{i}, \xi_{\mathcal{N}, T_{\delta}}^{k} \in S^{q}(Y, \mathcal{N})$ be the solutions of $(3.13)$ with right-hand side $l_{i}(\cdot), l_{k}(\cdot)$, respectively, as described above. Assume next that $S_{l}(Y)$ is an l-dimensional linear subspace of $S^{q}(Y, \mathcal{N})$ and consider $\xi_{l, T_{\delta}}^{i}, \xi_{l, T_{\delta}}^{k}$ the solution of (3.13) in $S_{l}(Y)$ with right-hand side $l_{i}(\cdot), l_{k}(\cdot)$, respectively. Define the following two numerical homogenized tensors

$$
\begin{aligned}
\left(a_{\mathcal{N}, T_{\delta}}^{0}\left(x_{\tau}\right)\right)_{i k} & =\int_{Y} a_{x_{\tau}}(y)\left(\nabla \hat{\xi}_{\mathcal{N}, T_{\delta}}^{i}(y)+\mathbf{e}_{\mathbf{i}}\right) \cdot\left(\nabla \hat{\xi}_{\mathcal{N}, T_{\delta}}^{k}(y)+\mathbf{e}_{\mathbf{k}}\right) d y \\
\left(a_{l, T_{\delta}}^{0}\left(x_{\tau}\right)\right)_{i k} & =\int_{Y} a_{x_{\tau}}(y)\left(\nabla \hat{\xi}_{l, T_{\delta}}^{i}(y)+\mathbf{e}_{\mathbf{i}}\right) \cdot\left(\nabla \hat{\xi}_{l, T_{\delta}}^{k}(y)+\mathbf{e}_{\mathbf{k}}\right) d y
\end{aligned}
$$

We first need the following lemma (see [10, Lemma 3.3]).

Lemma 3.2. Consider the tensors $a_{\mathcal{N}, T_{\delta}}^{0}\left(x_{\tau}\right), a_{l, T_{\delta}}^{0}\left(x_{\tau}\right)$ defined in (3.25), (3.26), respectively. Then

$$
\begin{aligned}
& \left|\left(a_{\mathcal{N}, T_{\delta}}^{0}\left(x_{\tau}\right)\right)_{i k}-\left(a_{l, T_{\delta}}^{0}\left(x_{\tau}\right)\right)_{i k}\right|= \\
& \left.\mid \int_{Y} a_{x_{\tau}}(y)\left(\nabla \hat{\xi}_{l, T_{\delta}}^{i}(y)-\nabla \hat{\xi}_{\mathcal{N}, T_{\delta}}^{i}(y)\right) \cdot\left(\nabla \hat{\xi}_{l, T_{\delta}}^{k}(y)-\nabla \hat{\xi}_{\mathcal{N}, T_{\delta}}^{k}(y)\right)\right) d y . \mid
\end{aligned}
$$

Proof. The proof follows the line of [10, Lemma 3.3]. We sketch it for completeness. As $S_{l}(Y) \subset S^{q}(Y, \mathcal{N})$ we have

$$
\begin{aligned}
& \left|\left(a_{\mathcal{N}, T_{\delta}}^{0}\left(x_{\tau}\right)\right)_{i k}-\left(a_{l, T_{\delta}}^{0}\left(x_{\tau}\right)\right)_{i k}\right| \\
& =\left|\int_{Y} a_{x_{\tau}}(y)\left(\nabla \hat{\xi}_{l, T_{\delta}}^{i}(y)-\nabla \hat{\xi}_{\mathcal{N}, T_{\delta}}^{i}(y)\right) \cdot \mathbf{e}_{\mathbf{k}} d y\right| \\
& =\left|\int_{Y} a_{x_{\tau}}(y)\left(\nabla \hat{\xi}_{l, T_{\delta}}^{i}(y)-\nabla \hat{\xi}_{\mathcal{N}, T_{\delta}}^{i}(y)\right) \cdot\left(\mathbf{e}_{\mathbf{k}}+\nabla \hat{\xi}_{l, T_{\delta}}^{k}(y)-\nabla \hat{\xi}_{\mathcal{N}, T_{\delta}}^{k}(y)\right) d y\right| \\
& =\left|\int_{Y} a_{x_{\tau}}(y)\left(\nabla \hat{\xi}_{l, T_{\delta}}^{i}(y)-\nabla \hat{\xi}_{\mathcal{N}, T_{\delta}}^{i}(y)\right) \cdot\left(-\nabla \hat{\xi}_{\mathcal{N}, T_{\delta}}^{k}(y)\right) d y\right|
\end{aligned}
$$

and the proof follows easily by further adding and subtracting the quantity $\nabla \hat{\xi}_{l, T_{\delta}}^{k}(y)$.

Notice that we have used the symmetry in the above proof. This proof is however valid without symmetry (following ideas in [12] or [32, Lemma 4.6], [33]). Next we derive an a 
posteriori estimator, which allows to control the accuracy of our output of interest (the numerically homogenized tensor). The procedure, which follows standard residual based estimates, is crucial for RB and has been extensively discussed (see [18] and [21] for a discussion in the homogenization context). Define

$$
\hat{e}_{l, T_{\delta}}^{i}=\hat{\xi}_{l, T_{\delta}}^{i}-\hat{\xi}_{\mathcal{N}, T_{\delta}}^{i} .
$$

Using (3.13) we see that

$$
b\left(\hat{e}_{l, T_{\delta}}^{i}, \hat{z}_{\mathcal{N}}\right)=b\left(\hat{\xi}_{l, T_{\delta}}^{i}, \hat{z}_{\mathcal{N}}\right)-l_{i}\left(\hat{z}_{\mathcal{N}}\right), \forall \hat{z}_{\mathcal{N}} \in S^{q}(Y, \mathcal{N}) .
$$

The right-hand side defines a linear form on $S^{q}(Y, \mathcal{N})$. Hence by the Riesz theorem, there exists a unique $\bar{e}_{l, T_{\delta}}^{i} \in S^{q}(Y, \mathcal{N})$ such that

$$
b\left(\hat{e}_{l, T_{\delta}}^{i}, \hat{z}_{\mathcal{N}}\right)=\left(\bar{e}_{l, T_{\delta}}^{i}, \hat{z}_{\mathcal{N}}\right)_{\mathcal{W}}
$$

where $(\cdot, \cdot)_{\mathcal{W}}$, defined as $(v, w)_{\mathcal{W}}=\int_{Y} \nabla v \cdot \nabla w d y$, denotes the scalar product in the space $W(Y)$ defined in 2.4 or 2.5). We notice that $\bar{e}_{l, T_{\delta}}^{i}$ can be computed numerically in an efficient way thanks to the affine representation of the tensor $a_{x_{\tau}}(y)$. This leads to define the residual of the a posteriori error estimator as

$$
\Delta_{l, T_{\delta}}^{i}:=\frac{\left\|\bar{e}_{l, T_{\delta}}^{i}\right\|_{\mathcal{W}}}{\sqrt{\lambda_{L B}}}
$$

Here $\lambda_{L B}$ is an approximation of the coercivity constant $\lambda$ described in 2.2 . To compute $\Delta_{l, T_{\delta}}^{i}$, one needs to solve (3.30), which is parameter dependent. Thanks to the affine representation of the tensor, 3.30 can be decomposed into several parameter independent FE problems that can be precomputed. Hence, the residual (3.31) is cheap to compute which is crucial to get the efficiency of the a posteriori control in the greedy algorithm (see [19, 20] for details). The next lemma gives the bound of the error in quantities of interest (e.g., the numerical homogenized tensors or the cell solutions) in terms of the residual (3.31).

Lemma 3.3. Let $\hat{\xi}_{l, T_{\delta}}^{i}, \hat{\xi}_{\mathcal{N}, T_{\delta}}^{i}$ be the solutions of problem 3.13 in $S_{l}(Y)$ and $S^{q}(Y, \mathcal{N})$, respectively, and $\bar{e}_{l, T_{\delta}}^{i}, \Delta_{l, T_{\delta}}^{i}, a_{\mathcal{N}, T_{\delta}}^{0}\left(x_{\tau}\right), a_{l, T_{\delta}}^{0}\left(x_{\tau}\right)$ be the quantities defined above. Assume that the approximation $\lambda_{L B}$ of the coercivity constant (2.2) satisfies $0<\lambda_{L B} \leq \lambda$. Then we have

$$
\begin{aligned}
& \left\|\hat{\xi}_{l, T_{\delta}}^{i}-\hat{\xi}_{\mathcal{N}, T_{\delta}}^{i}\right\|_{\mathcal{E}, K_{j}} \leq \Delta_{l, T_{\delta}}^{i}, \\
& \left\|\hat{\xi}_{l, T_{\delta}}^{i}-\hat{\xi}_{\mathcal{N}, T_{\delta}}^{i}\right\|_{\mathcal{W}} \leq \frac{\Delta_{l, T_{\delta}}^{i}}{\sqrt{\lambda_{L B}}}, \\
& \left.\left.\left(\frac{\lambda_{L B}}{\Lambda} \Delta_{l, T_{\delta}}^{i}\right)^{2} \leq \mid\left(a_{\mathcal{N}, T_{\delta}}^{0}\left(x_{\tau}\right)\right)\right)_{i i}-\left(a_{l, T_{\delta}}^{0}\left(x_{\tau}\right)\right)\right)_{i i} \mid \leq\left(\Delta_{l, T_{\delta}}^{i}\right)^{2},
\end{aligned}
$$

where $\Lambda$ is the continuity constant defined in $(2.2)$ and $\|\cdot\|_{\mathcal{E}, T_{\delta}}$ is the energy norm defined by

$$
\|v\|_{\mathcal{E}, T_{\delta}}=(b(v, v))^{1 / 2}:=\left(\int_{Y} a_{x_{\tau}}(y) \nabla v(y) \cdot \nabla v(y) d y\right)^{1 / 2}
$$


Proof. The proof is standard. Plugging $\hat{z}_{\mathcal{N}}=\hat{e}_{l, T_{\delta}}^{i}$ in 3.30 we get $\left\|\hat{e}_{l, T_{\delta}}^{i}\right\|_{\mathcal{E}, K_{j}}^{2}=\left(\bar{e}_{l, T_{\delta}}^{i}, \hat{e}_{l, T_{\delta}}^{i}\right)_{\mathcal{W}}$. On one hand, from the coercivity of (3.30) and the assumption on $\lambda_{L B}$ we get

$$
\left\|\hat{e}_{l, T_{\delta}}^{i}\right\|_{\mathcal{W}} \leq \frac{\left\|\bar{e}_{l, T_{\delta}}^{i}\right\|_{\mathcal{W}}}{\sqrt{\lambda_{L B}}}
$$

hence $(3.33)$. On the other hand, using the inequality $\sqrt{\lambda_{L B}}\left\|\hat{e}_{l, T_{\delta}}^{i}\right\|_{\mathcal{W}} \leq\left\|\hat{e}_{l, T_{\delta}}^{i}\right\|_{\mathcal{E}, T_{\delta}}$ easily leads to (3.32). The inequality (3.34) then follows from 3.27), 3.32 and the inequality $\left\|\bar{e}_{l, T_{\delta}}^{i}\right\|_{\mathcal{W}} \leq \Lambda\left\|\hat{e}_{l, T_{\delta}}^{i}\right\|_{\mathcal{W}}$.

Remark 3.4. From (3.34), we can see that the stability factor $\lambda_{L B}$ plays an important role in the efficiency of the a posteriori estimator. There are two efficient methods proposed in [34, 20, 19] to compute $\lambda_{L B}$. We simply mention them here (notice that for both methods, the affine representation (3.22) of the tensor is required). The simplest method is the "min $\Theta$ " method, where $\lambda_{L B}\left(x_{\tau}\right)$ is estimated by

$$
\lambda_{L B}\left(x_{\tau}\right)=\left(\min _{q \in\{1, \cdots, Q\}} \frac{\Theta_{q}\left(x_{\tau}\right)}{\Theta_{q}\left(\bar{x}_{\tau}\right)}\right) \lambda\left(\bar{x}_{\tau}\right),
$$

where $\bar{x}_{\tau}$ is a randomly chosen point in $\Omega$. The "min $\Theta$ " method, however, requires that the tensor (3.22) satisfies the following properties: (i) $\Theta_{q}(x)>0, q=1, \cdots, Q, \forall x \in \Omega$; (ii) $a_{q}(y) \xi \cdot \xi \geq 0, \forall \xi \in \mathbb{R}^{d}, y \in Y$. The above conditions might be restrictive for some applications. A more general but more involved method is the successive constraint method (SCM). This method is based on an offline-online strategy. The SCM offline stage relies on a greedy procedure and is costly, but the online procedure is very efficient. The advantage of this method is that it is a robust and general method which works for all kinds of affine tensors (we refer to [34, 20] for details).

Offline stage. We select by a greedy algorithm $N$ couple $\left(T_{\delta_{n}}, \eta_{n}\right)$, where $T_{\delta_{n}} \subset \Omega$ is a sampling domain and $\eta_{n}$ corresponds to the unit vector $\mathbf{e}_{\eta_{\mathbf{n}}}$ belonging to the set canonical basis of $\mathbb{R}^{d}$, where $\eta_{n} \in\{1, \ldots, d\}$. Corresponding to the $N$ couple $\left(T_{\delta_{n}}, \eta_{n}\right)$, we compute $\hat{\xi}_{\mathcal{N}, T_{\delta_{n}}}^{\eta_{n}}(\cdot)$, the solution of 3.13 with a tensor given by $a_{x_{\tau_{n}}}(y)\left(x_{\tau_{n}}\right.$ is the barycenter of $T_{\delta_{n}}$ ) and a right-hand side given by $l_{\eta_{n}}(\cdot)$. The following greedy algorithm to determine successively $\left\{\left(T_{\delta_{n}}, \eta_{n}, \hat{\xi}_{\mathcal{N}, T_{\delta_{n}}}^{\eta_{n}}\right), n=1, \ldots, N\right\}$ is based on the usual procedure of the RB methodology (see [18, 20]).

Algorithm 3.5 (Greedy procedure). Denote by $\|\cdot\|_{\mathcal{W}}$ the norm associated to the space $W(Y)$ (defined by (2.5) or (2.4)). Given two parameters, $N_{R B}$ the maximum basis number, and tol $_{R B}$ a stopping tolerance:

1. Choose randomly (by a Monte Carlo method) $N_{\text {train }}$ sampling domains $T_{\delta_{n}}$ in such a way that $T_{\delta_{n}} \subset \Omega$. Define the "training set" $\Xi_{R B}=\left\{\left(T_{\delta_{n}}, \eta_{n}\right) ; 1 \leq \eta_{n} \leq d, 1 \leq n \leq N_{\text {train }}\right\}$ '. $^{3}$.

\footnotetext{
${ }^{3} N_{\text {train }}$ should be large enough to ensure that the results of the greedy algorithm are stable with respect to other choices of training sets.
} 
2. Select randomly $\left(T_{\delta_{1}}, \eta_{1}\right) \in \Xi_{R B}$ and compute $\hat{\xi}_{\mathcal{N}, T_{\delta_{1}}}^{\eta_{1}}$, the solution of (3.13) with right-hand side $l_{\eta_{1}}(\cdot)$ in $S^{q}(Y, \mathcal{N})$, corresponding to the selected parameters $\left(T_{\delta_{1}}, \eta_{1}\right)$. Set $l=1$ and define $\hat{\xi}_{1, \mathcal{N}}(y)=\frac{\hat{\xi}_{\mathcal{N}, T_{\delta_{1}}}^{\eta_{1}}(y)}{\left\|\hat{\xi}_{\mathcal{N}, T_{\delta_{1}}}^{\eta_{1}}\right\|_{\mathcal{W}}}$, and the corresponding $R B$ space $S_{1}(Y)=\operatorname{span}\left\{\hat{\xi}_{1, \mathcal{N}}\right\}$.

3. For $l=2, \ldots, N_{R B}$

a. Compute for each $\left(T_{\delta}, \eta\right) \in \Xi_{R B}$ the solution $\hat{\xi}_{l-1, T_{\delta}}^{\eta}$ of 3.13 with right-hand side $l_{\eta}(\cdot)$ in $S_{l-1}(Y)$ and the residual $\Delta_{l-1, T_{\delta}}^{\eta}$ defined in 3.31 .

b. Select the next reduced basis by choosing

$$
\left(T_{\delta_{l}}, \eta_{l}\right)=\operatorname{argmax}_{\left(T_{\delta}, \eta\right) \in \Xi_{R B}} \Delta_{l-1, T_{\delta}}^{\eta}
$$

provided that $\max _{\left(T_{\delta}, \eta\right) \in \Xi_{R B}}\left(\Delta_{l-1, T_{\delta}}^{\eta}\right)^{2}>$ tol $_{R B}{ }^{4}$, otherwise the algorithm ends.

c. Compute $\hat{\xi}_{\mathcal{N}, T_{\delta_{l}}}^{\eta_{l}}$ the solution of 3.13 in $S^{q}(Y, \mathcal{N})$ corresponding to the selected parameters $\left(T_{\delta_{l}}, \eta_{l}\right)$. Set $\hat{\xi}_{l, \mathcal{N}}(y)=\frac{R_{l}(y)}{\left\|R_{l}(y)\right\|_{\mathcal{W}}}$ the $l-$ th $R B$ basis function, where

$$
R_{l}(y)=\hat{\xi}_{\mathcal{N}, T_{\delta_{l}}}^{\eta_{l}}(y)-\sum_{m=1}^{l-1}\left(\hat{\xi}_{\mathcal{N}, T_{\delta_{l}}}^{\eta_{l}}, \hat{\xi}_{m, \mathcal{N}}\right) \hat{\xi}_{m, \mathcal{N}}
$$

Define the $R B$ space $S_{l}(Y)=\operatorname{span}\left\{\hat{\xi}_{1, \mathcal{N}}, \ldots, \hat{\xi}_{l, \mathcal{N}}\right\}$. Set $l=l+1$ and go back to a.

We emphasize that while constructing $S_{l}(Y)$, with $\hat{\xi}_{l, \mathcal{N}}(y)$ being a linear combination of the solutions of $(3.13)$, our output of interest is (3.17) that can be computed using the RB (see Lemma 3.2). From Lemma 3.3, we know that the the square of the residual $\Delta_{l, T_{\delta}}^{\eta}$ gives an a posteriori error estimate for the output of interest, hence, $\left(\Delta_{l, T_{\delta}}^{\eta}\right)^{2}$ is the quantity that needs to be controlled in the above algorithm. We note that even though (3.13) has to be solved for each parameter in $\Xi_{R B}$ in the step 3.a., this procedure is moderately expensive as (3.13) is solved in the RB space $S_{l-1}(Y)$ of small dimension $l-1 \leq N$. A similar remark holds for the residuals $\Delta_{l, T_{\delta}}^{\eta}$ that need to be computed for each parameter in $\Xi_{R B}$, but only rely for these computations on precomputed quantities (computed once for the whole offline procedure) and small linear problems involving the current RB space $S_{l-1}(Y)$ (we again refer to [19, 20] for details).

Output of the offline procedure. The output of the above procedure is the RB space

$$
S_{N}(Y)=\operatorname{span}\left\{\hat{\xi}_{n, \mathcal{N}}(y), n=1, . ., N\right\}
$$

Rather than storing the reduced basis functions, using the affine representation (3.22) (or (3.23) ) described above, the output consists of the following matrices and vectors

$$
\left(A_{q}\right)_{n m}:=\int_{Y} a_{q}(y) \nabla \hat{\xi}_{n, \mathcal{N}}(y) \cdot \nabla \hat{\xi}_{m, \mathcal{N}} d y, \quad\left(F_{q}^{i}\right)_{m}=\int_{Y} a_{q}(y) \mathbf{e}_{\mathbf{i}} \nabla \hat{\xi}_{m, \mathcal{N}}(y) d y
$$

\footnotetext{
${ }^{4}$ Notice that the error of the outputs of interest scale like the square of the error of the cell functions as can be seen in Lemma 3.2 and 3.3 .
} 


\subsection{Online procedure and the RB-FE-HMM}

We define a macro method similar to the FE-HMM, with micro functions computed in the RB space. The method reads: find $u^{H, R B} \in S_{0}^{\ell}\left(\Omega, \mathcal{T}_{H}\right)$ such that

$$
B_{H, R B}\left(u^{H, R B}, v^{H}\right)=\int_{\Omega} f v^{H} d x, \forall v^{H} \in S_{0}^{\ell}\left(\Omega, \mathcal{T}_{H}\right)
$$

with a bilinear form given by

$$
B_{H, R B}\left(v^{H}, w^{H}\right):=\sum_{K \in \mathcal{T}_{H}} \sum_{j=1}^{J} \frac{\omega_{K_{j}}}{\left|K_{\delta_{j}}\right|} \int_{K_{\delta_{j}}} a^{\varepsilon}(x) \nabla v_{N, K_{j}}(x) \cdot \nabla w_{N, K_{j}}(x) d x,
$$

where $v_{N, K_{j}}(x)$ (respectively $w_{N, K_{j}}(x)$ ) is such that $v_{N, K_{j}}-v_{l i n, j}^{H}(x) \in S_{N}\left(K_{\delta_{j}}\right)$ and

$$
\int_{K_{\delta_{j}}} a^{\varepsilon}(x) \nabla v_{N, K_{j}}(x) \cdot \nabla z_{N}(x) d x=0, \quad \forall z_{N} \in S_{N}\left(K_{\delta_{j}}\right) .
$$

The space $S_{N}\left(K_{\delta_{j}}\right)$ is defined through the mapping $G_{x_{K_{j}}}: Y \rightarrow K_{\delta_{j}}$ as

$$
S_{N}\left(K_{\delta_{j}}\right)=\operatorname{span}\left\{\delta \hat{\xi}_{n, \mathcal{N}}\left(G_{x_{K_{j}}}^{-1}(x)\right)=: \xi_{n, K_{j}}(x), n=1, \ldots, N\right\} .
$$

The well-posedness of problem $\sqrt{3.39}$ is proved in the following lemma.

Lemma 3.6. Assume that (2.2) and that (Q1) hold. Then problem (3.39) has a unique solution.

Proof. Similarly as in [10], [9, Sect. 3.3.1] we can show that

$$
\left\|\nabla v_{\text {lin }}^{H}\right\|_{L^{2}\left(K_{\delta}\right)} \leq\left\|\nabla v_{N, K_{j}}\right\|_{L^{2}\left(K_{\delta}\right)} \leq \sqrt{\frac{\Lambda}{\lambda}}\left\|\nabla v_{l i n}^{H}\right\|_{L^{2}\left(K_{\delta}\right)}
$$

Using (2.2) and (Q1) we then obtain

$$
C_{1}\left\|\nabla v^{H}\right\|_{L^{2}(\Omega)}^{2} \leq B_{H, R B}\left(v^{H}, v^{H}\right), \quad B_{H, R B}\left(v^{H}, w^{H}\right) \leq C_{2}\left\|\nabla v^{H}\right\|_{L^{2}(\Omega)}\left\|\nabla w^{H}\right\|_{L^{2}(\Omega)} .
$$

The Poincaré inequality and the Lax-Milgram theorem give the stated result (see again [10], [9, Sect. 3.3.1] for details).

Fast solution of micro-problems. Owing to the affine form $(3.22)$ of the tensor $a^{\varepsilon}$, the problem (3.41) amounts to solving an $N \times N$ linear system (recall $N$ is small). Indeed, we observe that by writing $v_{N, K_{j}}-v_{l i n, j}^{H}(x)=\sum_{n=1}^{N} \alpha_{n} \xi_{n, K_{j}}(x)$ 3.41 reads

$$
\sum_{n=1}^{N} \alpha_{n} \int_{K_{\delta_{j}}} a^{\varepsilon}(x) \nabla \xi_{n, K_{j}}(x) \cdot \nabla \xi_{m, K_{j}}(x) d x=-\sum_{i=1}^{d} \int_{K_{\delta_{j}}} a^{\varepsilon}(x) \mathbf{e}_{\mathbf{i}} \cdot \nabla \xi_{m, K_{j}}(x) d x \frac{\partial v_{l i n, j}^{H}}{\partial x_{i}}
$$


for all $m=1, \ldots N$. Next, again thanks to the affine representation of the tensor (here we are assuming the representation (3.22) for simplicity), (3.44) can be written as

$$
\begin{aligned}
& \sum_{n=1}^{N} \alpha_{n} \sum_{q=1}^{Q} \Theta_{q}\left(x_{K_{j}}\right) \int_{Y} a_{q}(y) \nabla \hat{\xi}_{n, \mathcal{N}}(y) \cdot \nabla \hat{\xi}_{m, \mathcal{N}}(y) d y \\
= & -\sum_{i=1}^{d} \sum_{q=1}^{Q} \Theta_{q}\left(x_{K_{j}}\right) \int_{Y} a_{q}(y) \mathbf{e}_{\mathbf{i}} \cdot \nabla \hat{\xi}_{m, \mathcal{N}}(y) d y \frac{\partial v_{l i n, j}^{H}}{\partial x_{i}}
\end{aligned}
$$

or equivalently

$$
\left(\sum_{q=1}^{Q} \Theta_{q}\left(x_{K_{j}}\right) A_{q}\right) \boldsymbol{\alpha}=-\sum_{i=1}^{d}\left(\sum_{q=1}^{Q} \Theta_{q}\left(x_{K_{j}}\right) F_{q}^{i}\right) \frac{\partial v_{l i n, j}^{H}}{\partial x_{i}}
$$

where the $N \times N$ matrices $A_{q}, q=1, \ldots, Q$ and the vectors $F_{q}^{i} \in \mathbb{R}^{N}, q=1, \ldots, Q, i=$ $1, \ldots, d$ are defined by (3.38).

We emphasize that the matrices $A_{q}$ and the vectors $F_{q}^{i}$ are assembled and stored in the offline stage, thus (3.46) amounts just in building the linear combination by evaluating $\Theta_{q}(\cdot)$ at the desired integration points $x_{K_{j}}$ (or computing the interpolation problem (3.23) when we rely on the approximation $(3.23)$ for the tensor $\left.a_{x_{\tau}}(y)\right)$ and solving the $N \times N$ system (3.46) for each micro function at the quadrature points needed to assemble (2.7).

Reformulation of the RB-FE-HMM. Similar to the reformulation (3.16) for the FEHMM, we have

$$
B_{H, R B}\left(v^{H}, w^{H}\right):=\sum_{K \in \mathcal{T}_{H}} \sum_{j=1}^{J} \omega_{K_{j}} a_{N}^{0}\left(x_{K_{j}}\right) \nabla v_{l i n, j}^{H}\left(x_{K_{j}}\right) \cdot \nabla w_{l i n, j}^{H}\left(x_{K_{j}}\right),
$$

where

$$
\left(a_{N}^{0}\left(x_{K_{j}}\right)\right)_{i k}=\int_{Y} a_{x_{K_{j}}}(y)\left(\nabla \hat{\chi}_{N, K_{j}}^{i}(y)+\mathbf{e}_{\mathbf{i}}\right) \cdot\left(\nabla \hat{\chi}_{N, K_{j}}^{k}(y)+\mathbf{e}_{\mathbf{k}}\right) d y
$$

which is easily seen by noting that $v_{N, K_{j}}(x)$, the solution of 3.41 , can be written as

$$
v_{N, K_{j}}(x)=v_{l i n, j}^{H}(x)+\delta \sum_{i=1}^{d} \hat{\chi}_{N, K_{j}}^{i}\left(G_{x_{K_{j}}}^{-1}(x)\right) \frac{\partial v_{l i n, j}^{H}}{\partial x_{i}}
$$

where $\hat{\chi}_{N, K_{j}}^{i}(y)$ is the solution of 3.13 in the RB space 3.37).

\subsection{Reconstruction of the micro solution}

We briefly explain a procedure to obtain an approximation to the fine scale solution $u^{\varepsilon}$ of problem (2.1). While an error estimates $\left\|u^{\varepsilon}-u^{H, R B}\right\|$ can be obtained in the $L^{2}$ norm for locally periodic tensor (see Section 4), one cannot expect convergence between $\nabla u^{\varepsilon}$ and 
$\nabla u^{H, R B}$, as $u^{H, R B}$ does not capture the fine scale oscillations of the solution (we do not have strong convergence of $\nabla u^{\varepsilon}$ towards $\nabla u^{0}$ in general). Inspired by the correctors in homogenization theory [2], a numerical corrector for the FE-HMM has been introduced in [10, 26]. A numerical corrector, computed in a post-processing step can also be defined for the RB-FE-HMM. Here we present two approaches for such reconstruction.

For the first approach, which follows the procedure for the FE-HMM, we assume piecewise linear macro solver. For any $K \in \mathcal{T}_{H}$, we consider the function $u_{N, K}-u^{H, R B}$ known in the sampling domain $K_{\varepsilon}$ (see (3.41)). We then consider its periodic extension in $K$ denoted by $\left.\left(u_{N, K}-u^{H, R B}\right)\right|_{K} ^{P}$ and define a corrector in every macro element $K$ as

$$
\left.u_{p}(x)\right|_{K}=u^{H, R B}+\left.\left(u_{N, K}-u^{H, R B}\right)\right|_{K} ^{P} .
$$

An error estimate for this procedure is available for the FE-HMM in [10, Thm. 3.11] Also simple, this procedure requires to store the micro solutions in the sampling domains, and the periodic extension might be cumbersome, specially for three dimensional problems with simplicial elements. However, thanks to the precomputed RB space, the computational cost to solve a micro cell problem in the present framework is largely reduced. This allows to consider a second approach for the construction of numerical correctors. For a sampling domain of size $\varepsilon$, given $x \in K \in \mathcal{T}_{H}$, we can evaluate the reconstructed solution at this particular point by using

$$
\left.u_{p, R B}^{\varepsilon}(x)\right|_{K}=u^{H, R B}(x)+\varepsilon \sum_{i=1}^{d} \hat{\chi}_{N, T_{\varepsilon}}^{i}\left(G_{x}^{-1}(x)\right) \frac{\partial u_{l i n}^{H, R B}(x)}{\partial x_{i}},
$$

where $T_{\varepsilon}=x+(-\varepsilon / 2, \varepsilon / 2)^{d}$ and $\hat{\chi}_{N, T_{\varepsilon}}^{i}\left(G_{x}^{-1}(x)\right)$ can be computed by solving (3.13) in the reduced basis space $S_{N}\left(T_{\varepsilon}\right)$. We note that the second reconstruction procedure (3.51) allows to use higher order macro FEMs, whereas for the first procedure (3.50), it would require an interpolation procedure.

\section{A priori error analysis}

In this section, we derive an a priori error estimate for the RB-FE-HMM. While a component of the error (relying on the approximation property of the greedy algorithm) relies on assumptions difficult to check in practice, by providing the analysis proposed here, describing the various contributions to the global error is nevertheless of interest.

Following [9], an error estimate similar to (2.9) can be derived for the RB-FE-HMM.

Theorem 4.1. Let $u^{0}, u^{H, R B}$ be the solutions of (2.3) and (3.39), respectively, and that assume that $u^{0} \in \mathcal{H}^{l+1}(\Omega)$. Assume further that (Q1), (Q2) and (2.2) hold and that the tensor $a^{0}(x)$ appearing in (2.3) is sufficiently regular. Then

$$
\begin{aligned}
\left\|u^{0}-u^{H, R B}\right\|_{\mathcal{H}^{1}(\Omega)} & \leq C\left(H^{l}+r_{H M M}\right) \\
\left\|u^{0}-u^{H, R B}\right\|_{L^{2}(\Omega)} & \leq C\left(H^{l+1}+r_{H M M}\right)
\end{aligned}
$$


where

$$
r_{H M M}=\sup _{K_{H} \in \mathcal{T}_{H}, x_{K_{j}} \in K_{H}}\left\|a^{0}\left(x_{K_{j}}\right)-a_{N}^{0}\left(x_{K_{j}}\right)\right\|_{\mathcal{F}}
$$

and where the tensor $a_{N}^{0}$ is defined in (3.48) and $a^{0}\left(x_{K}\right)$ is the tensor of the homogenized problem (2.3) evaluated at the quadrature point $x_{K}$. The constant $C$ is independent of $H$, $N, \mathcal{N}$ or $\varepsilon$.

Proof. Decompose the error into $\left(\|\cdot\|\right.$ stands for the $L^{2}$ or $\mathcal{H}^{1}$ norm $)$

$$
\left\|u^{0}-u^{H, R B}\right\| \leq\left\|u^{0}-u^{0, H}\right\|+\left\|u^{0, H}-u^{H, R B}\right\|,
$$

where $u^{0, H}$ is the solution of (3.19).

Following [25], we obtain $\left\|u^{0}-u^{0, H}\right\|_{\mathcal{H}^{1}(\Omega)} \leq C H^{l}$ and $\left\|u^{0}-u^{0, H}\right\|_{L^{2}(\Omega)} \leq C H^{l+1}$ for sufficiently regular tensor $a^{0}$. We then have

$$
\left\|u^{0, H}-u^{H, R B}\right\|_{\mathcal{H}^{1}(\Omega)} \leq C \sup _{w^{H} \in S_{0}^{\ell}\left(\Omega, \mathcal{T}_{H}\right)} \frac{\left|B_{0, H}\left(u^{0, H}, w^{H}\right)-B_{H, R B}\left(u^{H, R B}, w^{H}\right)\right|}{\left\|w^{H}\right\|_{\mathcal{H}^{1}(\Omega)}} .
$$

Using the expressions (3.20) and (3.47) for $B_{0, H}$ and $B_{H, R B}$, respectively, together with (Q2), we can bound the right-hand side of the above inequality by $C \sup _{K_{H} \in \mathcal{T}_{H}} \sup _{x_{K} \in K_{H}} \| a^{0}\left(x_{K}\right)-$ $a_{N}^{0}\left(x_{K}\right)\left\|_{\mathcal{F}}\right\| u^{0, H} \|_{\mathcal{H}^{1}(\Omega)}$. Using the a priori bound $\left\|u^{0, H}\right\|_{\mathcal{H}^{1}(\Omega)} \leq C\|f\|_{\mathcal{H}^{-1}(\Omega)}$ completes the proof.

We further decompose

$$
r_{H M M} \leq r_{M O D}+r_{M I C}+r_{R B}
$$

with

$$
\begin{aligned}
& r_{M O D}:=\sup _{K_{H} \in \mathcal{T}_{H}, x_{K_{j}} \in K_{H}}\left\|a^{0}\left(x_{K_{j}}\right)-\bar{a}^{0}\left(x_{K_{j}}\right)\right\|_{\mathcal{F}}, \\
& r_{M I C}:=\sup _{K_{H} \in \mathcal{T}_{H}, x_{K_{j}} \in K_{H}}\left\|\bar{a}^{0}\left(x_{K_{j}}\right)-a_{\mathcal{N}}^{0}\left(x_{K_{j}}\right)\right\|_{\mathcal{F}}, \\
& r_{R B}:= \\
& \sup _{K_{H} \in \mathcal{T}_{H}, x_{K_{j}} \in K_{H}}\left\|a_{\mathcal{N}}^{0}\left(x_{K_{j}}\right)-a_{N}^{0}\left(x_{K_{j}}\right)\right\|_{\mathcal{F}},
\end{aligned}
$$

where $a_{\mathcal{N}}^{0}\left(x_{K_{j}}\right)$ is defined in (3.17) and $\bar{a}^{0}\left(x_{K_{j}}\right)$ is the tensor appearing in 3.18). Error bounds for the micro error $r_{M I C}$ were first presented in [10] for linear elliptic problems and generalized to high order in [9] (see also [29, Lemma 6]). Error bounds for the modeling error were first presented in [26] (see also [27] for a situation where $r_{M O D}$ vanishes). The aforementioned error estimates can directly be used for the RB-FE-HMM. It remains to estimate $r_{R B}$. Consider the space $\mathcal{M}^{\mathcal{N}}(Y)$ as defined in (3.21). We want to quantify how well $\mathcal{M}^{\mathcal{N}}(Y)$ can be approximated by the linear space $S_{N}(Y)$ of dimension $N$. Such a quantification relies on the notion Kolmogorov N-width. 
Definition 4.2. Let $F$ be a subset of $W(Y)$. We denote the distance of $F$ to any generic $N$-dimensional subspace $W_{N}(Y)$ of $W$ by

$$
E\left(F ; W_{N}\right)=\sup _{x \in F} \inf _{y \in W_{N}}\|x-y\|_{\mathcal{W}}
$$

The minimal error $E\left(F ; W_{N}(Y)\right)$ is given by the Kolmogorov n-width of $F$ in $W$

$$
d_{N}(F, W(Y))=\inf \left\{E\left(F ; W_{N}(Y)\right): W_{N}(Y) \text { a } N \text {-dimensional subspace of } W(Y)\right\} .
$$

It is difficult in general to quantify the Kolmogorov n-width of a given subset of $W(Y)$. Invoking regularity of the set $\mathcal{M}^{\mathcal{N}}(Y)$ with respect to the parameters one expects usually a fast (e.g. exponential) decay of $d_{N}$. Assuming such a decay, it is not obvious that the particular $N$-dimensional subspace of $W(Y)$ constructed with the greedy algorithm enjoys such an approximation property. This has been proved in [35, 36]. More precisely the application of [36, Corollary 3] shows the following result. Assume that the parametrized cell solution space $\mathcal{M}^{\mathcal{N}}$ has an exponentially small Komogorov n-width $d_{N}\left(\mathcal{M}^{\mathcal{N}}, W\right) \leq c e^{-r N}$, with $r$ satisfying

$$
r>\log \left(1+\left(\Lambda / \lambda_{L B}\right) \sqrt{\Lambda / \lambda}\right)
$$

where $\lambda, \Lambda$ are the coercivity and continuity bounds 2.2 and $\lambda_{L B}$ is the approximation of the coercivity constant used in the greedy algorithm (see Section 3.2). Then the reduced basis method converges exponentially in the sense that there exists a constant $s>0$ such that

$$
\left\|\hat{\chi}_{N, K_{j}}^{k}(y)-\hat{\chi}_{\mathcal{N}, K_{j}}^{k}(y)\right\|_{\mathcal{W}} \leq C e^{-s N}
$$

for all $K_{H} \in \mathcal{T}_{H}$ and all $x_{K_{j}} \in K_{H}$.

Theorem 4.3. In addition to the assumption of the Theorem 4.1, assume that the parametrized cell solution space $\mathcal{M}^{\mathcal{N}}$ has an exponentially small Komogorov n-width $d_{N}\left(\mathcal{M}^{\mathcal{N}}, W\right) \leq c e^{-r N}$, where $r$ satisfies 4.55. Then,

$$
\begin{aligned}
& \left\|u^{0}-u^{H, R B}\right\|_{\mathcal{H}^{1}(\Omega)} \leq C\left(H^{l}+e^{-2 s N}+r_{M I C}+r_{M O D}\right), \\
& \left\|u^{0}-u^{H, R B}\right\|_{L^{2}(\Omega)} \leq C\left(H^{l+1}+e^{-2 s N}+r_{M I C}+r_{M O D}\right) .
\end{aligned}
$$

If in addition $\chi^{i}, i=1, \ldots, d$, the solutions (3.12) in $W(Y)$ (see (2.5) (2.4) satisfy

$$
\left|\chi^{i}\right|_{\mathcal{H}^{q+1}\left(K_{\delta_{j}}\right)} \leq C \varepsilon^{-q} \sqrt{\left|K_{\delta_{j}}\right|}
$$

with $C$ independent of $\varepsilon$, the quadrature point $x_{K_{j}}$, then

$$
\begin{aligned}
\left\|u^{0}-u^{H, R B}\right\|_{\mathcal{H}^{1}(\Omega)} & \leq C\left(H^{l}+e^{-2 s N}+\left(\frac{h}{\varepsilon}\right)^{2 q}+r_{M O D}\right), \\
\left\|u^{0}-u^{H, R B}\right\|_{L^{2}(\Omega)} & \leq C\left(H^{l+1}+e^{-2 s N}+\left(\frac{h}{\varepsilon}\right)^{2 q}+r_{M O D}\right),
\end{aligned}
$$


where $\hat{h}=\frac{h}{\varepsilon}$ is the microscopic meshsize for the micro problems of the offline stage, i.e., $\hat{h}=\mathcal{O}\left(\mathcal{N}^{-1 / d}\right)$.

If in addition $a^{\varepsilon}(x)=a^{\varepsilon}(x, x / \varepsilon)=a(x, y)$ is $Y$-periodic in $y$, and $a_{i j}(x, y) \in \mathcal{C}\left(\bar{\Omega} ; \mathcal{W}_{\text {per }}^{1, \infty}(Y)\right)$ for all $i, j=1, \ldots, d$, then

$$
\begin{aligned}
& r_{M O D}=0 \quad \text { if } W\left(K_{\delta_{j}}\right)=\mathcal{W}_{p e r}^{1}\left(K_{\delta_{j}}\right) \text { and } \delta / \varepsilon \in \mathbb{N}, \\
& r_{M O D} \leq C\left(\delta+\frac{\varepsilon}{\delta}\right) \quad \text { if } W\left(K_{\delta_{j}}\right)=\mathcal{H}_{0}^{1}\left(K_{\delta_{j}}\right) \quad(\delta>\varepsilon) .
\end{aligned}
$$

Proof. The estimates 4.57, 4.58) follow from Theorem 4.1, 4.56) and Lemma 3.2, The estimates 4.60, 4.61) follow from Theorem 4.1, the estimates for the fully discrete error in [10] [28, Corollary 10] (see also [9, Lemma 10] and [29]). The estimate (4.63) has been proved in [26] and the estimate (4.62) in [27].

We notice that using the estimate $\left\|u^{\varepsilon}-u^{0}\right\|_{L^{2}(\Omega)} \leq C \varepsilon$ valid for locally periodic tensor [2, Chap. 1] we can obtain an error estimate

$$
\left\|u^{\varepsilon}-u^{H, R B}\right\|_{L^{2}(\Omega)} \leq C\left(H^{l+1}+e^{-2 s N}+\left(\frac{h}{\varepsilon}\right)^{2 q}+\varepsilon+r_{M O D}\right),
$$

measuring the approximation of $u^{H, R B}$ to the fine scale solution in the $L^{2}$ norm. In Theorem 4.3, various aspects of the convergence behavior of the RB-FE-HMM are described. The overall goal of the RB-FE-HMM is to obtain an approximation of the effective solution $u^{0}$. If the effective tensor would be available, then, rates of convergence such as $H^{\ell}$ or $H^{\ell+1}$ for the $\mathcal{H}^{1}$ or $L^{2}$ norm could be obtained, provided adequate regularity of the effective solution $u^{0}$. For effective solution with singularities, appropriate adaptive mesh refinement techniques could be used. In both case, the rate of convergence is described by classical FE analysis. To obtain similar convergence rates in terms of the macroscopic meshsize with the RB-FEHMM, we need to control the errors arising from the multiscale methodology and the use of micro solvers to recover the unknown effective data. These various errors are

- the microscopic error $\left(\frac{h}{\varepsilon}\right)^{2 q}$, where $\hat{h}=\frac{h}{\varepsilon}=\mathcal{O}\left(\mathcal{N}^{-1 / d}\right)$ is the microscopic meshsize and $q$ the order of the FEM used for the micro problems in the offline stage. In the RB framework, we use a very accurate meshsize for this offline problems, i.e., micro solvers with large value $\mathcal{N}$ of DOF. This effort is compensated by the fact that we solve micro problems only for a fixed (usually small number) of sampling domains distributed in the macroscopic physical computational, compared to the classical FE-HMM, where micro problems with DOF proportional to the macro DOF have to be solved in every macro elements (notice that in this case, not only the microscopic DOF but also the number of sampling domains increase while refining the macro mesh);

- the a priori RB error $e^{-2 s N}$, which quantifies how well the infinite dimensional manifold of solutions of micro problems can be approximated by a low dimensional linear subspace, explaining why a small number of micro problems usually suffice in the offline stage. In applications, however, the RB a priori estimate is not used (and usually not known), but this a priori error can be bounded (on both sides) by the RB a posteriori estimate which is computed during the RB procedure; 
- the modeling error, which indicates the influence of the micro solutions on the sampling domain sizes and and micro boundary conditions. In general, this is a delicate question already for the FE-HMM (without RB) and still a subject of investigation (see the discussion and references in the reviews [9, 29]). In particular cases, for example for locally periodic tensors with period $\varepsilon$, if we set the cell domain size $\varepsilon$ and choose the center of the cells at the quadrature points, then modeling error vanishes, i.e., $r_{M O D}=0$.

\section{Numerical examples}

In this section we apply the RB-FE-HMM to four test problems. The first three examples are 2D problems with an affine tensor, a discontinuous affine tensor and a nonaffine tensor, respectively. The fourth example is a $3 \mathrm{D}$ problem, representing heat transfer in a microchip first described in [37] for the FE-HMM. In the offline stage, the micro functions are computed in the reference domain $Y$ using a uniform mesh. We also use a uniform macroscopic mesh for the online procedure to compute the RB-FE-HMM solution. Notice that nonuniform meshes could be similarly used. For the 3D problem, we use the software CUBIT version 11.1 [38] to generate the macroscopic tetrahedral mesh for the discretization of the considered domain. Numerical evaluation of the errors. Let $u^{H}$ be the numerical solution and $u^{r e f}$ be a reference solution (for the problem (2.3)) computed on a fine triangulation $\mathcal{T}_{h}$. The error $u^{r e f}-u^{H}$ in the $\mathcal{H}^{1}$ and $L^{2}$ norms are estimated by

$$
\begin{aligned}
e_{L^{2}} & :=\left\|u^{h}\right\|_{L^{2}(\Omega)}^{-1}\left(\sum_{K \in \mathcal{T}_{h}} \sum_{j=1}^{J} w_{j, K}\left|u^{H}-u^{r e f}\right|\left(x_{K_{j}}\right)\right)^{-1 / 2} \\
e_{\mathcal{H}^{1}} & :=\left\|u^{h}\right\|_{\mathcal{H}^{1}(\Omega)}^{-1}\left(\sum_{K \in \mathcal{T}_{h}} \sum_{j=1}^{J} w_{j, K}\left|u^{H}-u^{r e f}\right|\left(x_{K_{j}}\right)\right)^{-1 / 2}
\end{aligned}
$$

where we will use $\|u\|_{\mathcal{H}^{1}(\Omega)} \sim\left(\sum_{K \in \mathcal{T}_{H}}\|\nabla u\|_{L^{2}(K)}^{2}\right)^{1 / 2}$. Here $\left\{x_{K_{j}}, w_{j, K}\right\}$ are quadrature points on the fine triangulation $\mathcal{T}_{h}$ chosen such that the quadrature formula is exact for the degree of the piecewise polynomials used to compute $u^{r e f}$.

Stability factor computation for the a posteriori error estimates. As explained in Remark (3.4), an estimation of the stability factor $\lambda_{L B}$ is crucial to control the accuracy of the outputs of the greedy algorithm. In the numerical experiments below, we will use the "min $\Theta$ " method when it can be applied (see Remark (3.4), namely for the 2D and 3D affine examples, and the SCM otherwise (for the 2D discontinuous and nonaffine examples).

\subsection{2-dimensional problems.}

Let $\Omega=[0,1]^{2}$. We consider the following problem

$$
\begin{aligned}
-\nabla \cdot\left(a^{\varepsilon}(x) \nabla u^{\varepsilon}(x)\right) & =1, & & \text { in } \Omega, \\
u^{\varepsilon}(x) & =0, & & \text { on } \partial \Omega_{D}, \\
n \cdot\left(a^{\varepsilon}(x) \nabla u^{\varepsilon}(x)\right) & =0, & & \text { on } \partial \Omega_{N},
\end{aligned}
$$


where for $x=\left(x_{1}, x_{2}\right), \partial \Omega_{D}=\left\{x_{1}=0\right\} \cup\left\{x_{1}=1\right\}$ and $\partial \Omega_{N}=\left\{x_{2}=0\right\} \cup\left\{x_{2}=1\right\}$. We will choose various oscillating tensors $a^{\varepsilon}$ for the above problem. The tensors are chosen so that the homogenized tensors can be easily computed to be able to perform careful numerical test on the behavior of the experimental convergence of the RB-FE-HMM. We emphasize that our numerical method can be applied to more general problems, when an explicit form of the homogenized tensor is not available (e.g., non-periodic or random tensors).

$2 \mathrm{D}$ affine multiscale tensor. We consider

$a\left(x, \frac{x}{\varepsilon}\right)=\left(\begin{array}{cc}x_{1}^{2}+0.2+\left(x_{2}+1\right)\left(\sin \left(2 \pi \frac{x_{1}}{\varepsilon}\right)+2\right) & 0 \\ 0 & x_{2}^{2}+0.05+\left(x_{1} x_{2}+1\right)\left(\sin \left(2 \pi \frac{x_{2}}{\varepsilon}\right)+2\right)\end{array}\right)$,

with a corresponding homogenized tensor given by

$$
a^{0}(x)=\left(\begin{array}{cc}
\left(\int_{0}^{1} \frac{1}{x_{1}^{2}+0.2+\left(x_{2}+1\right)\left(\sin \left(2 \pi y_{1}\right)+2\right)} d y_{1}\right)^{-1} & 0 \\
0 & \left(\int_{0}^{1} \frac{1}{x_{2}^{2}+0.05+\left(x_{1} x_{2}+1\right)\left(\sin \left(2 \pi y_{2}\right)+2\right)} d y_{2}\right)^{-1}
\end{array}\right)
$$

In the offline stage, we use the P1-FEM and the P2-FEM respectively, to compute the $\mathrm{RB}$ for the micro problems, that is, we take the FE space $S^{q}(Y, \mathcal{N})$ with $q=1$, 2 , with a large number of DOF (as usual in the RB methodology). Periodic coupling is used (i.e., $S^{q}(Y, \mathcal{N})$ is chosen to be a subspace of (2.4)). We also choose sampling domains that match the length of the period of $a^{\varepsilon}$. To make a fair comparison, we take the same initial sample set $\Xi_{R B}$ for the computations with the P1 and the P2 FEM. In both cases, the tolerance, set

Table 1: Parameters for the offline stage (affine tensor).

\begin{tabular}{l|c|c}
\hline Offline stage & P1-FEM & P2-FEM \\
\hline Meshsize for the micro reference domain $Y$ & $1500 \times 1500$ & $1200 \times 1200$ \\
Initial sample points number $\Xi_{R B} \subset \Omega$ & 800 & 800 \\
Tolerance for the offline stage $t o l_{R B}$ & $5 \mathrm{e}-11$ & $5 \mathrm{e}-11$ \\
Stability factor method & $\min \Theta$ & $\min \Theta$ \\
\hline RB number & 10 & 10 \\
Final a posteriori error & $1.7059 \mathrm{e}-12$ & $2.7682 \mathrm{e}-11$ \\
Offline CPU time(s) & 5330 & 42724 \\
\hline
\end{tabular}

to $\operatorname{tol}_{R B}=5 \mathrm{e}-11$, is reached by the a posteriori estimator after the selection of 10 reduced basis. The CPU time in second is reported for our MATLAB computations. We see that the P2 FEM is approximately 8 times more expensive than the P1 FEM for the offline stage. In Fig. 3, we report the decay of the a posteriori error $\left(\Delta_{l, T_{\delta}}^{\eta}\right)^{2}$ that is fast, as expected.

With these precomputed RB spaces of micro solutions (obtained with P1 and P2 FEM), we now perform online computation and compute the macro solution $u^{H, R B}$ with the RBFE-HMM. We use P1, P2 and P3 macro FEMs to compute $u^{H, R B}$. The macro meshsize is chosen as $2^{-n}, n=3, \ldots, 8$. A reference solution $u^{r e f}$ is computed by solving (2.3) with a tensor given by (5.65) using a FEM with a $1024 \times 1024$ mesh and piecewise polynomials of 
total degree 3. The behavior of the error of the RB-FE-HMM is shown in Fig. 4. In view of the estimates of Theorem 4.3, we can make the following observations. First, we notice that $r_{M O D}=0$ as we have periodic boundary conditions for the RB-FE-HMM and sampling domains of size $\varepsilon$. According to the a priori estimates 4.61), 4.60), the micro error $r_{M I C}$

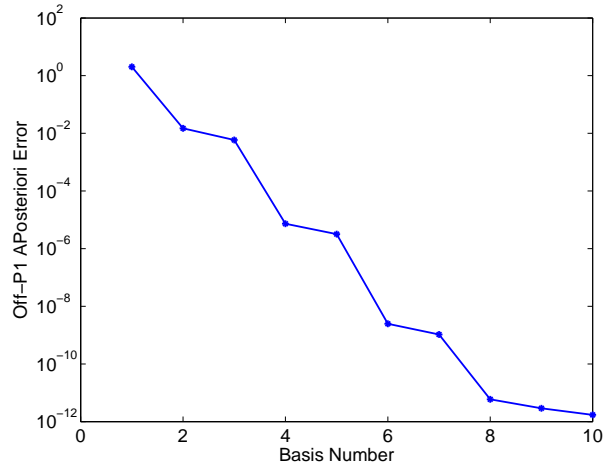

(a) P1-Offline A posteriori Error

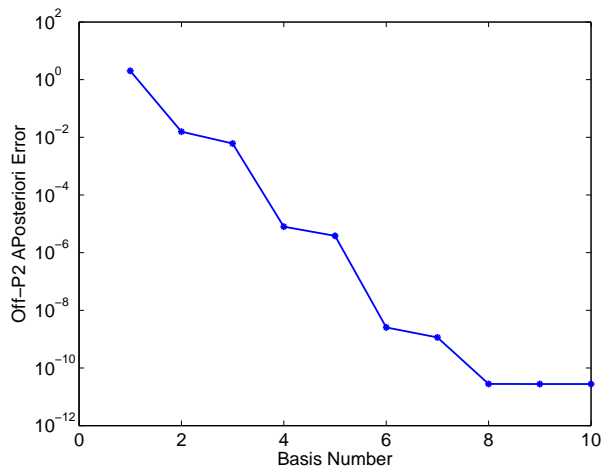

(b) P2-Offline A posteriori Error

Fig. 3: Affine tensor a posteriori error $\max _{\left(T_{\delta}, \eta\right) \in \Xi_{R B}}\left(\Delta_{l, T_{\delta}}^{\eta}\right)^{2}$.

should be of the order of $10^{-7}, 10^{-13}$ for the P1, P2 offline computation, respectively, while the macro error should be of the order $2^{-n \cdot(P+1)}, 2^{-n \cdot P}$ for the $L^{2}$ and $\mathcal{H}^{1}$ errors, respectively, where $\mathrm{P}$ is the order of the macro FEM and $n=3, \ldots 8$. For the computations with $\mathrm{P} 1$ polynomials in the offline stage, the results are in accordance with the theoretical results. Whenever the macro error reaches $10^{-7}$, we lose the expected convergence rates which may indicate that micro errors are of the same order of accuracy (this can be seen in Fig. 4 (a) $-(\mathrm{b}))$.

For the computation with P2 polynomials in the offline stage, one would expect the effect of micro errors appearing for macro errors around $10^{-13}$, but we see in Fig. 4 (c) that the expected convergence rates are lost for errors around $10^{-11}$. Here we think that what is seen is the $r_{R B}$ error term, i.e., the accuracy of the reduced basis procedure. Indeed, according to the a posteriori error estimator (controlling this latter error), the accuracy reached for P2 polynomials in the offline procedure with the given mesh is around $10^{-11}$ (see Table (1)).

How does the RB-FE-HMM compare with the FE-HMM? We next show some comparisons with $u^{H, R B}$ computed with P1 offline and online polynomials.

We choose two different offline meshes, namely $350 \times 350$ and $500 \times 500$ and obtain 10 RB (as previously). For the FE-HMM solution $u^{H}$, we use P1-FEM for both the macro and micro solvers, where simultaneous refinement is needed according to estimates (2.9), (2.10). The errors in the $L^{2}$ norm and the computation time are reported in Tables 2 (error) and 3 (computation time). In Table 2, we see that the offline mesh of size $350 \times 350$ is fine enough to get the optimal (quadratic) convergence rate. The simultaneous refinement for the FE-HMM, i.e., $H \simeq(h / \varepsilon) \simeq 2^{-n}, n=3, \ldots 8$, also gives the optimal (quadratic) convergence rate. The errors for both methods are similar. Now we compare in Table 3 


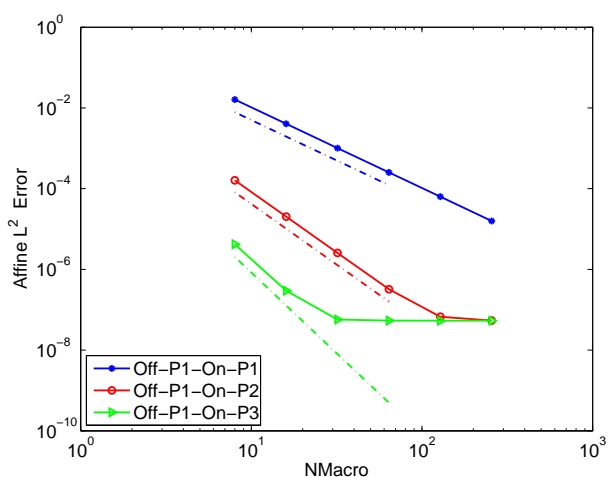

(a) Offline P1-FEM $L^{2}$ Error

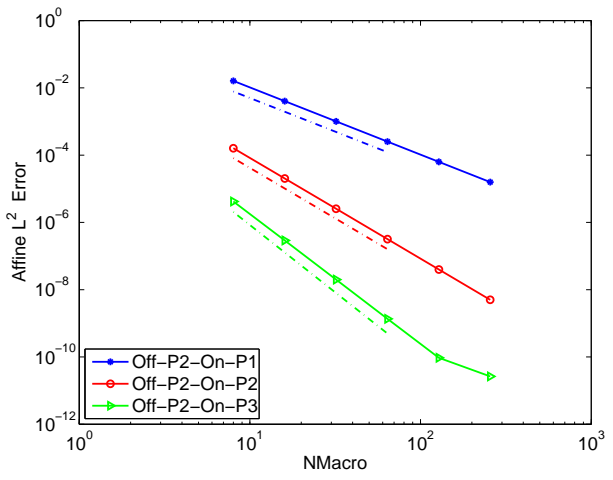

(c) Offline P2-FEM $L^{2}$ Error

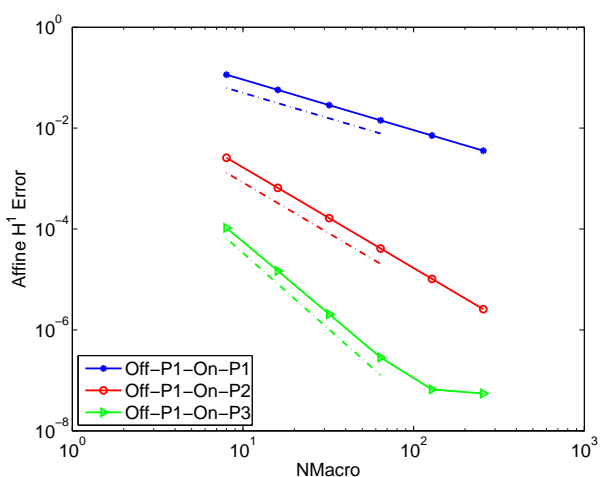

(b) Offline P1-FEM $\mathcal{H}^{1}$ Error

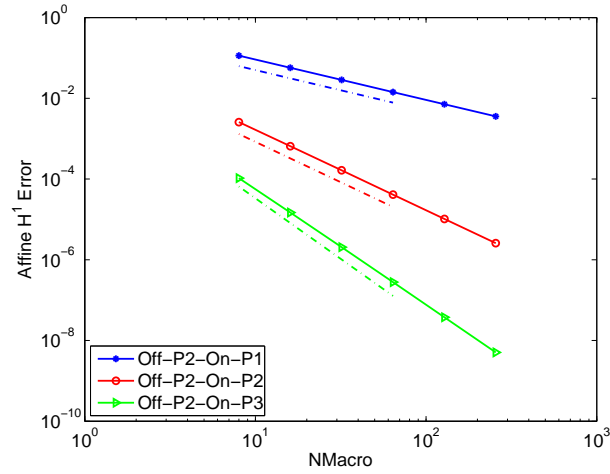

(d) Offline P2-FEM $\mathcal{H}^{1}$ Error

Fig. 4: Affine tensor example $\left\|u^{H, R B}-u^{r e f}\right\|$. The offline and RB space info is in Table 1 . Online solver is P1-FEM, P2-FEM, P3-FEM respectively. The dashed lines are the reference lines with slopes 2,3,4 for (a)-(c) and slopes $1,2,3$ for (b)-(d).

Table 2: Comparison between RB-FE-HMM (P1-FEM as offline and online solver) and FE-HMM (P1-FEM as micro and macro solver) for the $L^{2}$ error.

\begin{tabular}{c|c|c|c}
\hline & $\begin{array}{c}\text { RB-FE-HMM } \\
\text { offline mesh } 350 \times 350 \\
L^{2} \text { Error }\end{array}$ & $\begin{array}{c}\text { RB-FE-HMM } \\
\text { offline mesh } 500 \times 500\end{array}$ & FE-HMM \\
\hline $8 \times 8$ & 0.0161 & $L^{2}$ Error & $L^{2}$ Error \\
\hline $16 \times 16$ & 0.0040 & 0.0161 & 0.0176 \\
$32 \times 32$ & 0.0010 & 0.0040 & 0.0044 \\
$64 \times 64$ & $2.5347 \mathrm{e}-04$ & 0.0010 & 0.0011 \\
$128 \times 128$ & $6.3969 \mathrm{e}-05$ & $2.5306 \mathrm{e}-04$ & $2.7702 \mathrm{e}-04$ \\
$256 \times 256$ & $1.6599 \mathrm{e}-05$ & $6.3561 \mathrm{e}-05$ & $6.9259 \mathrm{e}-05$ \\
\hline
\end{tabular}

the computation time. Taking into account the offline stage, we see that the total cost is 
Table 3: Comparison of CPU time between the RB-FE-HMM (P1-FEM as the offline and online solver) and the FE-HMM (P1-FEM as the micro and macro solver). The offline CPU time is $193 s$ with meshsize $350 \times 350$ and $424 s$ with meshsize $500 \times 500$.

\begin{tabular}{c|c|c}
\hline & $\begin{array}{c}\text { RB-FE-HMM } \\
\text { Online CPU Time (s) } \\
\text { with 1 processor }\end{array}$ & $\begin{array}{c}\text { FE-HMM } \\
\text { CPU Time (s) } \\
\text { with 1 processor }\end{array}$ \\
\hline $8 \times 8$ & 0.03 & 0.14 \\
$16 \times 16$ & 0.10 & 0.98 \\
$32 \times 32$ & 0.28 & 109 \\
$64 \times 64$ & 1.21 & 1760 \\
$128 \times 128$ & 4.92 & 27504 \\
$256 \times 256$ & 20.33 & 332410 \\
\hline
\end{tabular}

an order of magnitude smaller for the RB-FE-HMM except for very coarse macro meshes, where overhead given by the cost of the offline computation for the RB-FE-HMM dominates the cost for the FE-HMM. As can be seen from these computations, for errors smaller than $10^{-4}$, the RB-FE-HMM is always more efficient than the FE-HMM.

Notice that in our comparisons between the FE-HMM and the RB-FE-HMM we only used P1 online macro FEMs. Because of the increasing number of cell problems that need to be solved for the FE-HMM when using higher order macro polynomials (due to the increasing number of quadrature points and related sampling domains), this method becomes very expensive. In contrast, only the macro assembly is affected in the RB-FE-HMM (similarly as for standard FEM) when using higher order macro solver. Thus for the RB-FE-HMM the cost of increasing the degree of the macro polynomials is proportional to the macro DOFs only and is similar to the cost of increasing the polynomials degree in standard FEM.

2D affine multiscale discontinuous tensor. In this example, we test the RB-FE-HMM on a problem with an oscillating tensor discontinuous on the sampling domains. Such tensors prevent the use of fast microsolvers (e.g., based on pseudo-spectral methods as proposed in [11]). This is why we distinguish in our experiments continuous versus discontinuous affine tensors. We assume that the reference domain is divided into three subdomains $Y=$ $Y_{A} \cup Y_{B} \cup Y_{C}$ with different tensors in the different domains, discontinuous at the interfaces. The tensor is defined by

$$
a\left(x, \frac{x}{\varepsilon}\right)=a_{A}\left(x, \frac{x}{\varepsilon}\right) I_{A}+a_{B}\left(x, \frac{x}{\varepsilon}\right) I_{B}+a_{C}\left(x, \frac{x}{\varepsilon}\right) I_{C},
$$

where $I_{A}, I_{B}, I_{C}$ are the indicator functions of the domains $Y_{A}, Y_{B}, Y_{C}$ and $a_{A}\left(x, \frac{x}{\varepsilon}\right), a_{B}\left(x, \frac{x}{\varepsilon}\right)$, 
$a_{C}\left(x, \frac{x}{\varepsilon}\right)$ are diagonal tensors with entries given by

$$
\begin{aligned}
& a_{A, i i}\left(x, \frac{x}{\varepsilon}\right)= \begin{cases}x_{1}^{2}+0.2+\left(x_{2}+1\right)\left(\sin \left(2 \pi \frac{x_{1}}{\varepsilon}\right)+2\right), & i=1 \\
x_{2}^{2}+0.05+\left(x_{1} x_{2}+1\right)\left(\sin \left(2 \pi \frac{x_{2}}{\varepsilon}\right)+2\right), & i=2\end{cases} \\
& a_{B, i i}\left(x, \frac{x}{\varepsilon}\right)= \begin{cases}3 x_{1}+x_{2}^{2}+0.5, & i=1 \\
e^{\left(-x_{1}-x_{2}\right)}\left(\cos \left(2 \pi \frac{x_{1}}{\varepsilon}\right)+2\right), & i=2\end{cases} \\
& a_{C, i i}\left(x, \frac{x}{\varepsilon}\right)=x_{1}+x_{2}+1, i=1,2 .
\end{aligned}
$$

Notice that the above tensor could model a material having different phases with different conductivity properties in each phase. The discontinuities over the phases are illustrated in Fig. 5 (b)-(c). Table 4 provides information of the offline stage.

Table 4: Parameters for RB-FE-HMM offline stage (discontinuous tensor).

\begin{tabular}{c|c}
\hline Meshsize for the micro reference domain $Y$ & $901 \times 901$ \\
\hline Initial sample points number $\Xi_{R B} \subset \Omega$ & 800 \\
Tolerance of the offline stage $t o l_{R B}$ & $1 \mathrm{e}-07$ \\
Offline solver & P1-FEM \\
Method used to compute the stability factor & SCM \\
RB number & 40 \\
\hline
\end{tabular}

For this example, we use the FE-HMM solution with fine micro and macro meshes to compute a reference solution (we choose a grid of $512 \times 512$ for the micro and macro meshes, respectively). We display in Fig. 6 (a) the RB-FE-HMM solution with macro mesh $128 \times 128$. In Fig. 6 (b) we report the $L^{2}$ and $\mathcal{H}^{1}$ convergence rates (as we refine the macro mesh for the RB-FE-HMM). As can be seen, we obtain optimal convergence rates for this example. This shows the efficiency of the RB strategy in situations (discontinuous tensors) that prevent the use of fast micro solvers taking advantage of the smoothness of the micro solution.

2D nonaffine multiscale tensor. For the last 2-dimensional example, we consider a tensor that is not in affine form. As mentioned in Section 3.2, we have to apply the EIM to obtain an affine representation of the tensor to implement the RB methodology. We take a tensor of the form

$$
\begin{aligned}
& a\left(x, \frac{x}{\varepsilon}\right)_{11}=\left(\sqrt{\left(x_{1}^{2}+\sin \left(2 \pi \frac{x_{1}}{\varepsilon}\right)+1.2\right)\left(x_{1} x_{2}+\sin \left(4 \pi \frac{x_{1}}{\varepsilon}\right)+1.5\right)}\right)^{-1} \\
& a\left(x, \frac{x}{\varepsilon}\right)_{22}=\left(\left(x_{1} x_{2}+\sin \left(5 \pi \frac{x_{2}}{\varepsilon}\right)+1.2\right)\left(x_{2}^{2} \cos \left(2 \pi \frac{x_{2}}{\varepsilon}\right)+x_{1}+1.5\right)\right)^{-1} \\
& a\left(x, \frac{x}{\varepsilon}\right)_{12}=a\left(x, \frac{x}{\varepsilon}\right)_{21}=0
\end{aligned}
$$

chosen in such a way that the homogenized tensor can be computed easily for numerical 


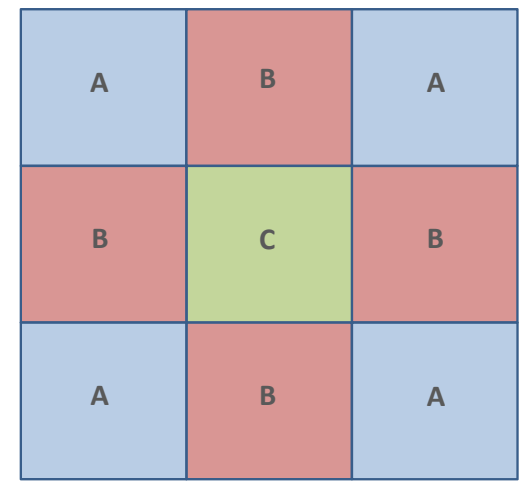

(a) The substructure of the micro reference domain.
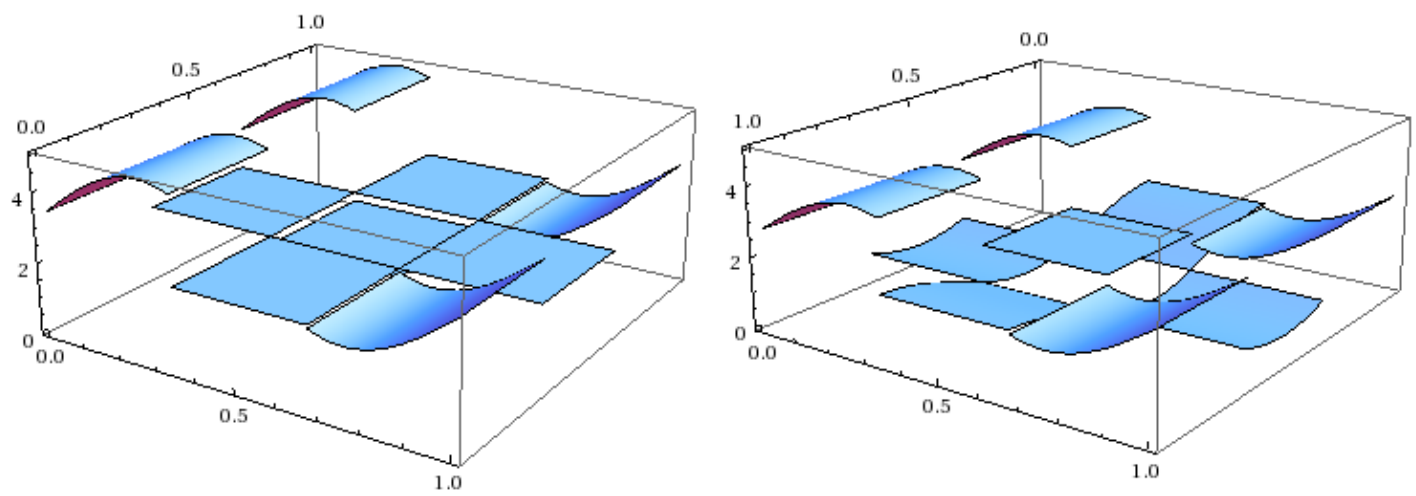

(b) Upper diagonal term of $a\left(x, \frac{x}{\varepsilon}\right)=a(x, y)$ (c) Lower diagonal term of $a\left(x, \frac{x}{\varepsilon}\right)=a(x, y)$ with $\left(x_{1}, x_{2}\right)=(0.5,0.5)$. with $\left(x_{1}, x_{2}\right)=(0.5,0.5)$

Fig. 5: Domain and tensor. 


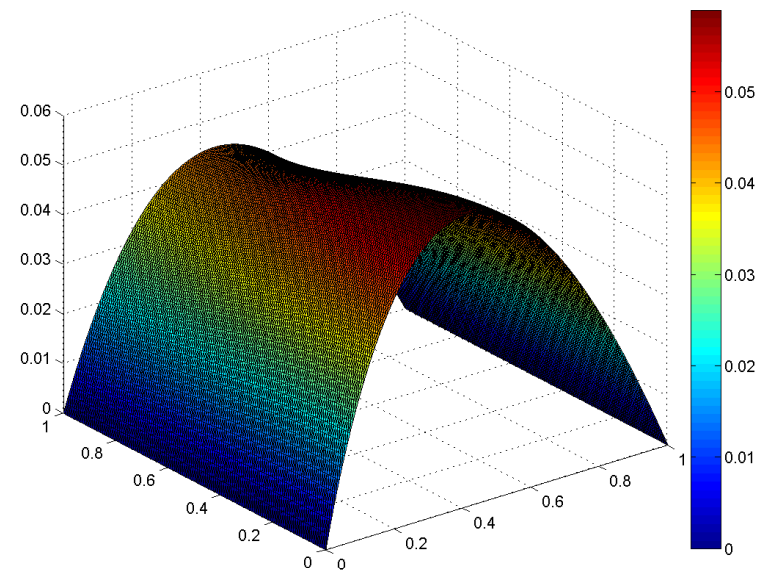

(a) $u^{H, R B}$

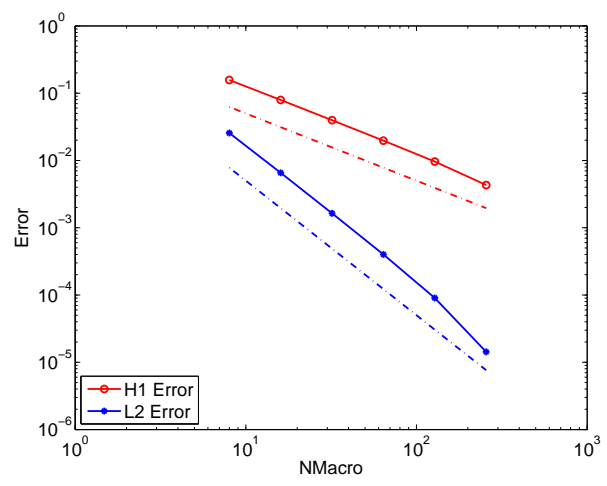

(b) $\left\|u^{H, R B}-u^{r e f}\right\|$

Fig. 6: RB-FE-HMM for discontinuous tensor. (a). RB-FE-HMM solution computed with a $128 \times 128$ online macro mesh. (b). The error $\left\|u^{H, R B}-u^{r e f}\right\|$ is displayed, where 40 reduced bases are used for $u^{H, R B}$. The online solver is a P1-FEM. The reference solution $u^{\text {ref }}$ is computed by the FE-HMM with $512 \times 512$ micro and macro meshes. The dashed lines are the reference lines with the slope 1,2 , respectively.

comparison purpose. It is given by

$$
\begin{aligned}
& a_{11}^{0}=\left(\int_{0}^{1} \sqrt{\left(x_{1}^{2}+\sin \left(2 \pi y_{1}\right)+1.2\right)\left(x_{1} x_{2}+\sin \left(4 \pi y_{1}\right)+1.5\right)} d y_{1}\right)^{-1} \\
& a_{22}^{0}=\left(\int_{0}^{1}\left(x_{1} x_{2}+\sin \left(5 \pi y_{2}\right)+1.2\right)\left(x_{2}^{2} \cos \left(2 \pi y_{2}\right)+x_{1}+1.5\right) d y_{2}\right)^{-1} \\
& a_{12}^{0}=a_{21}^{0}=0
\end{aligned}
$$

In Table 5 we report the parameters of the EIM offline stage. We obtained 19 affine terms for the first diagonal entry of $a\left(x, \frac{x}{\varepsilon}\right)$, and 26 terms for the second diagonal entry.

Table 5: Parameters for EIM offline stage.

\begin{tabular}{l|c}
\hline Initial sample points number $\Xi_{E I M} \subset \Omega$ & 600 \\
Tolerance of the EIM tol & $1 \mathrm{e}-06$ \\
Number affine terms for $\left(a\left(x, \frac{x}{\varepsilon}\right)\right)_{11}$ & 19 \\
Number affine terms for $\left(a\left(x, \frac{x}{\varepsilon}\right)\right)_{22}$ & 26 \\
EIM CPU time(s) & 5461 \\
\hline
\end{tabular}

Next, we report the parameters of the RB-FE-HMM offline stage in Table 6. Since the error from the EIM process also influences the output accuracy of the offline stage, we choose a lower accuracy requirement for the offline stage and fix $t o l_{R B}$ to be $10^{-8}$. This tolerance is met by the the a posteriori error estimator of the greedy procedure after the selection of 13 
Table 6: Parameters for the RB-FE-HMM offline stage.

\begin{tabular}{l|c}
\hline Meshsize for the micro reference domain $Y$ & $1200 \times 1200$ \\
\hline Initial sample points number $\Xi_{R B} \subset \Omega$ & 800 \\
Tolerance of the offline stage $\operatorname{tol}_{R B}$ & $1 \mathrm{e}-08$ \\
Offline solver & P1 FEM \\
Method used to compute the stability factor & SCM \\
RB number & 13 \\
Offline CPU time (s) & 57354 \\
\hline
\end{tabular}

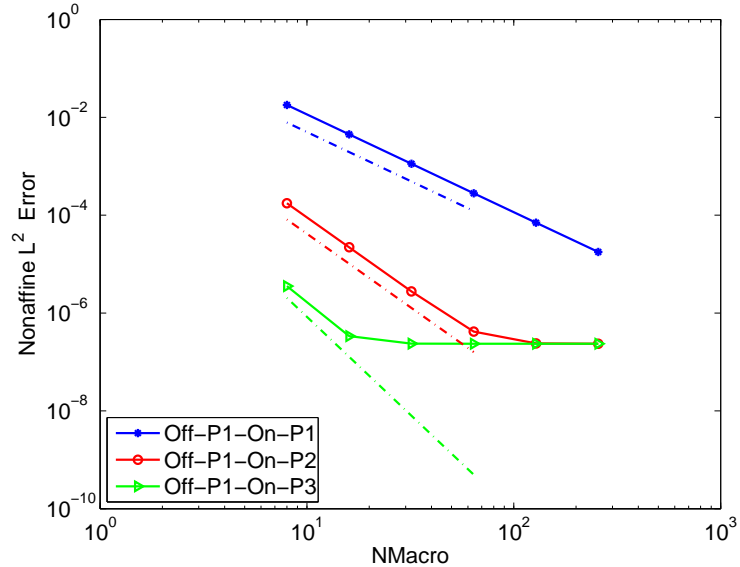

(a) $L^{2}$ Error

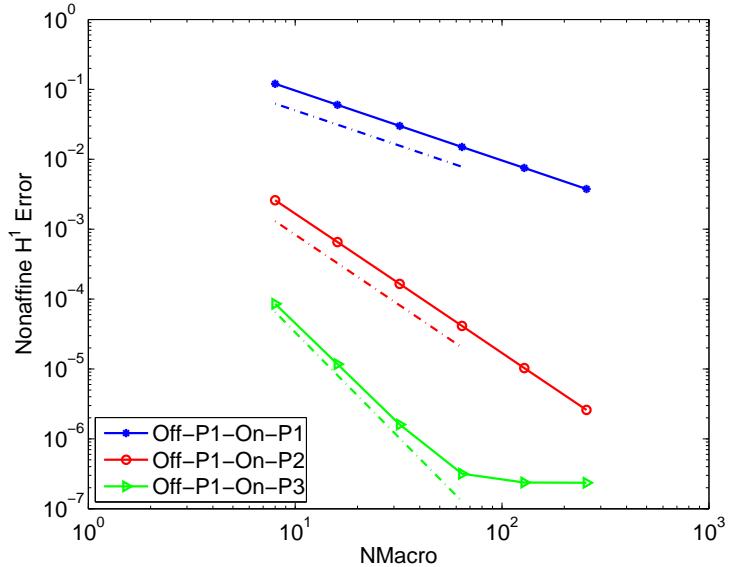

(b) $\mathcal{H}^{1}$ Error

Fig. 7: Problem with nonaffine tensor. The error $\left\|u^{H, R B}-u^{r e f}\right\|$ is displayed, where 13 reduced bases are used for computing $u^{H, R B}$. The offline parameters are reported in Table 6 . For the online macro solver P1-FEM, P2-FEM, P3-FEM are used. The dashed lines are the reference lines with slope equal to 2,3,4 in Fig. (a) and 1,2,3 in Fig. (b). The homogenized FE solution $u^{\text {ref }}$ is computed with a P3-FEM on a fine mesh of size $1024 \times 1024$.

bases. As we fix the tolerance at $10^{-8}$, we do not expect to get optimal convergence rates when the error is smaller than this threshold. Observe that here, an additional error term should appear in the $r_{H M M}$ error described in Theorem 4.1, namely the approximation error due to the EIM. Experimentally we observe a plateau when the error reaches $10^{-7}$. Until this threshold, we observe in Fig. 7 optimal convergence for the RB-FE-HMM (with P1, P2 or P3 macro FEMs). Let us mention that the tensor chosen here is continuous and that the performance of the EIM may decrease when the coefficients vary discontinuously within a sampling domain (see [22, Chapter 5]).

\subsection{Three-dimensional test problem.}

The FE-HMM, as any numerical homogenization methods, can be costly for threedimensional problems, due to the repeated computations of micro problems on sampling 
domains, each of them involving an increasing number of DOF as the mesh on the computational domain is refined (remember that this requires the micro mesh on the sampling domain to be refined simultaneously to the macro mesh).

The problem considered here is the heat transfer in a microchip (see Fig 8), as described in [37. The volume of the smallest box containing the microchip is $12.2 \times 12.2 \times 1 \mathrm{~mm}^{3}$. The macro domain $\Omega$ is composed of three parts, $\Omega=\Omega_{\text {chip }} \cup \Omega_{\text {leadframe }} \cup \Omega_{\text {resin }}$, the domains

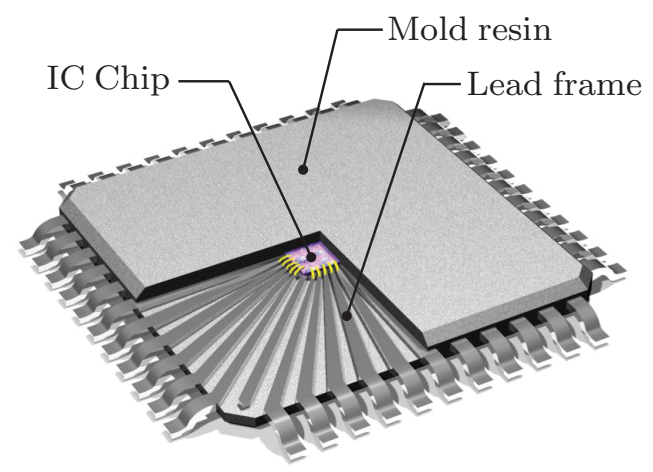

Fig. 8: 3D Macro domain structure [37].

of the chip, the leadframe and the package, respectively (see Fig. 8). The model equation is given by

$$
\begin{aligned}
-\nabla \cdot\left(a^{\varepsilon} \nabla u^{\varepsilon}\right) & =f \quad \text { in } \Omega, \\
n \cdot\left(a^{\varepsilon} u^{\varepsilon}\right)+\alpha u^{\varepsilon} & =g_{R} \quad \text { on } \partial \Omega,
\end{aligned}
$$

where $g_{R}=5863\left[\frac{W}{m^{2}}\right]$ and $\alpha=20$ and

$$
f=\left\{\begin{array}{cc}
1.87 \times 10^{8}\left[\frac{\mathrm{W}}{\mathrm{m}^{3}}\right] & x \in \Omega_{\text {chip }} \\
0 & \text { otherwise }
\end{array}\right.
$$

Here $g_{R}$ represents the heat flux entering the domain and the heat exchange with the ambient temperature and $f$ is a heat source representing the power of the chip. We take different conductivity tensors for each component. All the tensors are diagonal and given by

$$
\begin{aligned}
& a_{i i, c h i p}^{\varepsilon}=140, \\
& a_{i i, \text { leadframe }}^{\varepsilon}=400 e^{20\left(x_{1}^{2}+x_{2}^{2}\right)^{\frac{1}{2}}}+400\left(\cos \left(x_{3} \pi\right)+1.5\right)\left(\cos \left(2 \pi x_{i} / \varepsilon\right)+1.1\right) \text {, } \\
& a_{i i, \text { resin }}^{\varepsilon}= \begin{cases}\frac{1}{\sin \left(6 \pi x_{2}\right)+1.6}+3\left(\cos \left(\pi x_{3}\right)+1.5 x_{1}^{2}+1.1\right)\left(\sin \left(4 \pi x_{1} / \varepsilon\right)+1.1\right) & i=1, \\
\frac{1}{\sin \left(6 \pi x_{2}\right)+1.6}+3\left(\cos \left(\pi x_{3}\right)+1.5 x_{1}^{2}+1.1\right)\left(\cos \left(\pi x_{2} / \varepsilon\right)+1.2\right) & i=2, \\
\frac{2}{\sin \left(6 \pi x_{2}\right)+1.6}+3\left(\cos \left(\pi x_{3}\right)+1.5 x_{1}^{2}+1.1\right)\left(\cos \left(6 \pi x_{3} / \varepsilon\right)+1.2\right) & i=3 .\end{cases}
\end{aligned}
$$

We notice that $a_{c h i p}^{\varepsilon}$ is constant and we do not need to solve any cell problem on the domain $\Omega_{\text {chip }}$. We thus apply the RB-FE-HMM strategy on $\Omega_{\text {leadframe }}$ and $\Omega_{\text {resin }}$ respectively. 
Table 7: 3D RB-FE-HMM offline parameters.

\begin{tabular}{|c|c|c|c|c|}
\hline Domain & Initial sample points number & Offline mesh & tol $_{R B}$ & RB number \\
\hline$\Omega_{\text {leadframe }}$ & 400 & $220 \times 220 \times 220$ & $1 \mathrm{e}-10$ & 11 \\
$\Omega_{\text {resin }}$ & 600 & $220 \times 220 \times 220$ & $1 \mathrm{e}-10$ & 9 \\
\hline
\end{tabular}

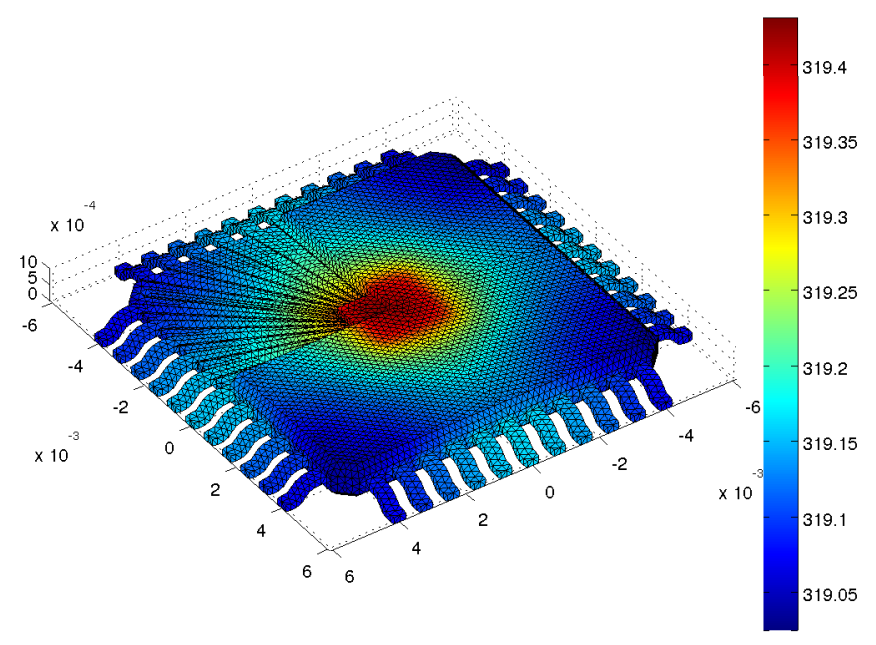

Fig. 9: 3D RB-FE-HMM solution $u^{H, R B}$. The offline parameters are reported in Table 7 Online DOF: 37011. 
Table 7 displays the RB offline parameters. We see that 20 reduced bases are needed for this problem.

A solution of problem (5.66) computed with the RB-FE-HMM is shown in Fig. 9. For this computation a macro mesh with 37011 DOF is used. In Table 8, we show error estimates when comparing the RB-FE-HMM solution $u^{H, R B}$ with a numerically computed reference solution $u^{r e f}$ for the homogenized problem. For $3 \mathrm{D}$ problems, it is not a trivial task to compute an accurate reference solution. The reference solution is computed as follows. As $a_{\text {leadframe }}^{\varepsilon}$ and $a_{\text {resin }}^{\varepsilon}$ are diagonal tensors, the corresponding homogenized tensors $a_{i i \text {,leadframe }}^{0}$ and $a_{i i \text {,resin }}^{0}$ are the harmonic averages of the fine scale tensors. These harmonic averages (involving 1D integrals) are further evaluated by using an accurate numerical integration scheme.

Because of the difficulty to obtain an accurate reference solution, we only display the accuracy of the RB-FE-HMM for one refinement step. A refinement step (corresponding roughly to a meshsize divided by two), starting with the initial mesh with 37011 DOF (corresponding to 190081 tetrahedra), leads to 278123 DOF (corresponding to 1520648 tetrahedra). The reference solution is computed with a mesh with 2108977 DOF (corresponding to 12165184 tetrahedra). We display in Table 8 the error in various norms, when we refine the macro mesh. We see that the $\mathcal{H}^{1}$ and the $L^{2}$ errors have the expected decay rate.

Table 8: Error estimates for problem (5.66). The solution $u^{\text {ref }}$ is computed with $2108977 \mathrm{DOF},\left\|u^{\text {ref }}\right\|_{A}=$ 24.2616 .

\begin{tabular}{c|c|c|c|c}
\hline Online mesh DOF & $\left\|u^{H, R B}\right\|_{A}$ & $\left\|u^{H, R B}-u^{r e f}\right\|_{L^{2}(\Omega)}$ & $\left\|u^{H, R B}-u^{r e f}\right\|_{\mathcal{H}^{1}(\Omega)}$ & $\frac{\left\|u^{H, R B}-u^{r e f}\right\|_{L^{\infty}(\Omega)}}{\left\|u^{r e f}\right\|_{L^{\infty}(\Omega)}}$ \\
\hline 37011 & 24.2465 & 0.0001665 & 0.0214941 & 0.0002173 \\
278123 & 24.2604 & $5.6277 \mathrm{e}-05$ & 0.0081573 & $7.9756 \mathrm{e}-05$ \\
\hline
\end{tabular}

We emphasize that once the reduced 20 bases are computed in the offline stage, the online stage is quite inexpensive. In contrast, computations with the FE-HMM would require to solve a very large number of cell problems on sampling domains (about 1.5 million for the mesh with $278123 \mathrm{DOF}$ ), with a meshsize adapted to the rate of decay of the macro meshsize. For a macro mesh that is not overly coarse (this is already required to properly represent the geometry of the microchip), a computation with the FE-HMM is much more costly than with the RB-FE-HMM, even for a single computation when taking into account the offline and the online costs for this latter method. For such 3D problems it is thus very advantageous to use the RB-FE-HMM even for computation with low order (piecewise linear) macro FEMs. 
Conclusion. We have presented an efficient FEM for high order discretizations of elliptic homogenization problems based on micro-macro solvers combined with a RB strategy. In our new method, the RB-FE-HMM, repeated FEM computations of micro problems (at quadrature points of a macro mesh) are avoided. These repeated micro computations are the main computational overhead of a numerical homogenization method such as the FEHMM, when accurate macro solutions need to be computed. In turn, the RB-FE-HMM is not only more efficient for high order macroscopic disretizations, but also for three-dimensional problems, already for low order macroscopic discretizations when even a single micro problem in each element of the macroscopic mesh is expensive to compute. Using interpolation techniques following the reduced basis methodology, we showed that an efficient numerical method can be designed, relying only on a small number of accurately computed micro solutions. An a posteriori error estimate for the selection of representative micro solutions has been discussed. We have derived an a priori error analysis which allows to describe the decay rate of the various discretization errors involved in our numerical approach. The efficiency of the RB-FE-HMM strategy has been illustrated by several numerical examples and comparisons with the classical FE-HMM have shown significant improvements.

Acknowledgements. This work was supported in part by the Swiss National Science Foundation under Grant 200021 134716/1.

\section{References}

[1] A. Bensoussan, J.-L. Lions, G. Papanicolaou, Asymptotic analysis for periodic structures, North-Holland Publishing Co., Amsterdam, 1978.

[2] V. Jikov, S. Kozlov, O. Oleinik, Homogenization of differential operators and integral functionals, Springer-Verlag, Berlin, Heidelberg, 1994.

[3] T. Hou, X. Wu, Z. Cai, Convergence of a multiscale finite element method for elliptic problems with rapidly oscillating coefficients, Math. Comp. 68 (227) (1999) 913-943.

[4] Y. Efendiev, T. Y. Hou, Multiscale finite element methods. Theory and applications, vol. 4 of Surveys and Tutorials in the Applied Mathematical Sciences, Springer, New york, 2009.

[5] J. Oden, S. Prudhomme, A. Romkes, P. Bauman, Multiscale modeling of physical phenomena: adaptive control of models, SIAM J. Sci. Comput. 28 (6) (2006) 2359-2389.

[6] V. H. Hoang, christoph Schwab, High-dimensional finite elements for elliptic problems with multiple scales, Multiscale Model. Simul 3 (1) (2005) 168-194.

[7] M. Geers, V. Kouznetsova, W. Brekelmans, Multi-scale computational homogenization: Trends and challenges, J. Comput. Appl. Math. 234 (2010) 2175-2182.

[8] W. E, B. Engquist, The heterogeneous multiscale methods, Commun. Math. Sci. 1 (1) (2003) 87-132. 
[9] A. Abdulle, The finite element heterogeneous multiscale method: a computational strategy for multiscale PDEs, GAKUTO Int. Ser. Math. Sci. Appl. 31 (2009) 135-184.

[10] A. Abdulle, On a priori error analysis of fully discrete heterogeneous multiscale FEM, SIAM, Multiscale Model. Simul. 4 (2) (2005) 447-459.

[11] A. Abdulle, B. Engquist, Finite element heterogeneous multiscale methods with near optimal computational complexity, SIAM, Multiscale Model. Simul. 6 (4) (2007) 10591084 .

[12] R. Du, P. Ming, Heterogeneous multiscale finite element method with novel numerical integration schemes, Commm. Math. Sci. 8 (4) (2010) 863-885.

[13] A. Abdulle, A. Nonnenmacher, A posteriori error analysis of the heterogeneous multiscale method for homogenization problems, C. R. Math. Acad. Sci. Paris 347 (17-18) (2009) 1081-1086.

[14] A. Abdulle, A. Nonnenmacher, Adaptive finite element heterogeneous multiscale method for homogenization problems, Comput. Methods Appl. Mech. Engrg. 200 (3740) (2011) 2710-2726.

[15] R. L. Fox, H. Miura, An approximate analysis technique for design calculations, AIAA Journal 9 (1) (1971) 177-179.

[16] J. P. Fink, W. C. Rheinboldt, On the error behavior of the reduced basis technique for nonlinear finite element approximations, Z. Angew. Math. Mech. 63 (1983) 21-28.

[17] A. K. Noor, Recent advances in reduction methods for nonlinear problems, Comput. Struct. 13 (1981) 31-44.

[18] C. Prud'homme, D. V. Rovas, K. Veroy, L. Machiels, Y. Maday, A. T. Patera, G. Turinici, Reliable real-time solution of parametrized partial differential equations: Reduced-basis output bounds methods, J. Fluids Eng. 124 (2002) 70-80.

[19] A. T. Patera, G. Rozza, Reduced Basis Approximation and A Posteriori Error Estimation for Parametrized Partial Differential Equations, to appear in (tentative rubric) MIT Pappalardo Graduate Monographs in Mechanical Engineering, 2007.

[20] G. Rozza, D. Huynh, A. T. Patera, Reduced basis approximation and a posteriori error estimation for affinely parametrized elliptic coercive partial differential equations, Arch. Comput. Methods. Eng. 15 (2008) 229-275.

[21] S. Boyaval, Reduced-Basis approach for homogenization beyond the periodic setting, Multiscale Model. Simul. 7 (1) (2008) 466-494.

[22] S. Boyaval, Mathematical modeling and simulation for material science, $\mathrm{PhD}$ thesis, University Paris Est, 2009. 
[23] N. C. Nguyen, A multiscale reduced-basis method for parametrized elliptic partial differential equations with multiple scales, J. Comp. Phys. 227 (2008) 9807-9822.

[24] L. Tartar, Estimations des coefficients homogénéisés, Lectures Notes in Mathematics 704, Springer-Verlag, Berlin, 1977.

[25] P. Ciarlet, P. Raviart, The combined effect of curved boundaries and numerical integration in isoparametric finite element methods, Math. Foundation of the FEM with Applications to PDE (1972) 409-474.

[26] W. E, P. Ming, P. Zhang, Analysis of the heterogeneous multiscale method for elliptic homogenization problems, J. Amer. Math. Soc. 18 (1) (2005) 121-156.

[27] A. Abdulle, C. Schwab, Heterogeneous Multiscale FEM for diffusion problems on rough surfaces, SIAM, Multiscale Model. Simul. 3 (1) (2005) 195-220.

[28] A. Abdulle, Discontinuous Galerkin finite element heterogeneous multiscale method for elliptic problems with multiple scales, Math. Comp. 81 (278) (2012) 687-713.

[29] A. Abdulle, A priori and a posteriori error analysis for numerical homogenization: a unified framework, Ser. Contemp. Appl. Math. CAM 16 (2011) 280-305.

[30] M. Barrault, Y. Maday, N. Nguyen, A. Patera, An 'empirical interpolation method': Application to efficient reduced-basis discretization of partial differential equations, $\mathrm{C}$. R. Acad. Sci. Paris Ser.I 339 (2004) 667-672.

[31] Y. Maday, N. C. Nguyen, A. T. Patera, G. Pau, A general multipurpose interpolation procedure: the magic points, Commun. Pure Appl. Anal. 8 (1) (2009) 383-404.

[32] A. Abdulle, G. Vilmart, Analysis of the finite element heterogeneous multiscale method for nonmonotone elliptic homogenization problems, preprint submitted for publication.

[33] A. Abdulle, G. Vilmart, The effect of numerical integration in the finite element method for nonmonotone nonlinear elliptic problems with application to numerical homogenization methods, C. R. Acad. Sci. Paris, Ser. I 349 (19-20) (2011) 1041-1046.

[34] D. Huynh, G. Rozza, S. Sen, A. Patera, A successive constraint linear optimaization method for lower bounds of parametric coercivity and inf-sup stability constants, C. R. Math. Acad. Sci. Paris, Ser.I 345 (2007) 473-478.

[35] P. Binev, A. Cohen, W. Dahmen, R. Devore, G. Petrova, P. Wojtaszczyk, Convergence rates for greedy algorithms in reduced basis methods, preprint .

[36] A. Buffa, Y. Maday, A. T. Patera, C. Prud'homme, G. Turinici, A priori convergence of the greedy algorithm for the parametrized reduced basis, to appear in ESAIM-Math. Model. Numer. Anal. Special Issue in honor of David Gottlieb. 
[37] A. Abdulle, A. Nonnenmacher, A short and versatile finite element multiscale code for homogenization problem, Comput. Methods Appl. Mech. Engrg 198 (37-40) (2009) 2839-2859.

[38] Sandia National Lab, http://cubit.sandia.gov, 1997-2010. 